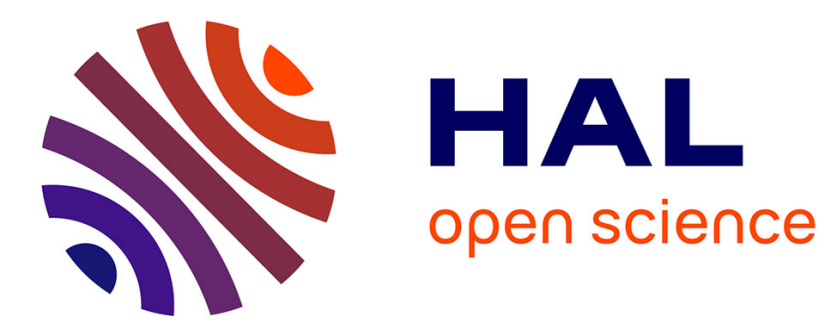

\title{
Effective Dynamic Properties of a Row of Elastic Inclusions: The Case of Scalar Shear Waves
}

\author{
Jean-Jacques Marigo, Agnes Maurel, Kim Pham, Amine Sbitti
}

\section{To cite this version:}

Jean-Jacques Marigo, Agnes Maurel, Kim Pham, Amine Sbitti. Effective Dynamic Properties of a Row of Elastic Inclusions: The Case of Scalar Shear Waves. Journal of Elasticity, 2017, 128 (2), pp.265-289. 10.1007/s10659-017-9627-4 . hal-01657090

\section{HAL Id: hal-01657090 \\ https://hal-polytechnique.archives-ouvertes.fr/hal-01657090}

Submitted on 7 Dec 2017

HAL is a multi-disciplinary open access archive for the deposit and dissemination of scientific research documents, whether they are published or not. The documents may come from teaching and research institutions in France or abroad, or from public or private research centers.
L'archive ouverte pluridisciplinaire HAL, est destinée au dépôt et à la diffusion de documents scientifiques de niveau recherche, publiés ou non, émanant des établissements d'enseignement et de recherche français ou étrangers, des laboratoires publics ou privés. 


\title{
Effective dynamic properties of a row of elastic inclusions : the case of scalar shear waves
}

\author{
Jean-Jacques Marigo • Agnès Maurel • \\ Kim Pham • Amine Sbitti
}

Received : date / Accepted : date

Résumé We present the homogenization of a periodic array of elastic inclusions embedded in an elastic matrix. We consider shear elastic waves with a typical wavelength $1 / k$ much larger than the array spacing $h$ and thickness $e$. Owing to the small parameter $\eta=k h$, with $e / h=O(1)$, a matched asymptotic expansion technique is applied to the wave equation in the time domain. The homogenized problem involves an equivalent interface associated to jump conditions of the Ventcels type. Up to the accuracy of the model in $O\left(\eta^{2}\right)$, different jump conditions are possible, which correspond to enlarged versions of the interface; these jump conditions are parametrized by the thickness $a$ of the homogenized interface. We inspect the influence of $a$ (i) on the equation of energy conservation in the homogenized problem and (ii) on the error of the model for a simple scattering problem. We show that restoring the thickness of the real array, $a=e$, is the optimal configuration regarding both aspects.

Keywords shear waves · interface homogenization · Matched asymptotic expansion

\section{Introduction}

In previous papers, we addressed the problem wave propagation through thin arrays of periodic cracks or voids, which are associated to Neumann boun-

J.-J. Marigo

Lab. de Mécanique des Solides, Ecole Polytechnique, Route de Saclay, Palaiseau, France

E-mail: marigo@lms.polytechnique.fr

A. Maurel

Inst. Langevin, ESPCI, 1 rue Jussieu, Paris, France, E-mail: agnes.maurel@espci.fr

K. Pham

UME, 828, bd des Maréchaux, Palaiseau, France E-mail: kim.pham@ensta-paristech.fr

A. Sbitti

École Normale Supérieure de l'Enseignement Technique, B.P. 6207 Rabat, Maroc 


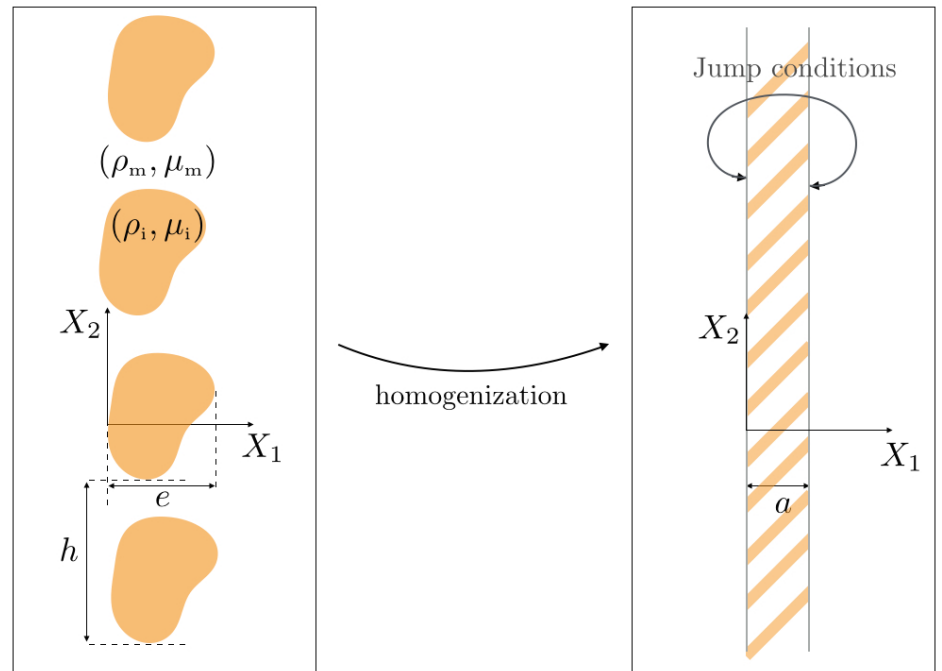

Figure 1 The array of inclusions in the matrix, with thickness $e$, are replaced by a layer of thickness $a$ across which jump conditions apply.

dary condition on the boundaries of the cracks. This was done in the context of acoustics for sound hard inclusions [1,2] and in the context of electromagnetism for metallic inclusions [3, 4, where the Neumann boundary conditions also apply. Thanks to the small parameter $\eta \equiv k h=O(k e)$, with $k$ the typical wavenumber imposed by the wave source and $h, e$ the array spacing and the array thickness, a homogenization method was applied, yielding an equivalent problem where the array is replaced by a zero thickness interface across which jump conditions apply, and the model is developed up to order $O\left(\eta^{2}\right)$. These jump conditions can be rewritten in different forms being all equivalent up to $O\left(\eta^{2}\right)$ when considering a non zero thickness interface, with a thickness artificially set to some $a=O(h, e)$ value. The different forms of the jump conditions have been discussed in 5, 6 in terms of the stability of the obtained conditions (with respect to $\eta$ and with respect to the frequency) and models up to $O\left(\eta^{3}\right)$ were considered in these references. In a different perspective, when numerical implementation of the homogenized problem in the time domain is sought, a negative interface energy is not acceptable since it produces instabilities in time [11. In [1,2,3 and in [11, we followed the intuitive argument that the equivalent interface has to occupy the same region as the original array, and we used $a=e$.

In the present paper, we extend these works, where cracks (or their acoustic and electromagnetic counterparts) were considered, to the case of elastic inclusions supporting wave propagation. We restrict our study to the scalar case of shear waves, and at some point to rectangular inclusions, which allows for explicit calculations. Next, the influence of the thickness $a$ of the enlarged interface is considered in terms (i) of the condition under which the interface energy is positive and (ii) of the accuracy of the homogenized solution with 
respect to the solution of the actual problem. The paper is organized as follow. In Section 2, we derive the effective jumps of the displacement and of the normal stress across the equivalent interface with arbitrary thickness $a$, Eqs. (42), which involve effective parameters being dependent of $a$, Eqs. (40). The equation of energy conservation is obtained, Eq. (44), with a contribution due to the non smoothness of the fields and which corresponds to the energy supported by the homogenized interface. In Section 3, we discuss the influence of $a$ on the accuracy of the homogenized solution with respect to the exact one, that is computed in the real problem and on the sign of the interface energy. Our findings show that $a=e$ is indeed the best choice, which reduces the discrepancy between the homogenized and the actual solutions and which ensures a positive energy supported by the interface.

\section{The interface model}

We start with the wave equation for the elastic displacement $U(\mathbf{X}, t)$ and stress vector $\boldsymbol{\Sigma}(\mathbf{X}, t)$ associated to shear waves, written in the time domain

$$
\rho \frac{\partial^{2} U}{\partial t^{2}}=\operatorname{div} \boldsymbol{\Sigma}, \quad \boldsymbol{\Sigma}=\mu \boldsymbol{\nabla} U
$$

with time $t$ and spatial coordinate $\mathbf{X}=\left(X_{1}, X_{2}\right) ; \rho$ denotes the mass density and $\mu$ the shear modulus, both being spatially dependent. (1) can be written using $V \equiv \partial U / \partial t$

$$
\left\{\begin{array}{l}
\frac{\partial \boldsymbol{\Sigma}}{\partial t}=\mu \boldsymbol{\nabla} V \\
\rho \frac{\partial V}{\partial t}=\operatorname{div} \boldsymbol{\Sigma} .
\end{array}\right.
$$

Next, in a bounded domain $\mathcal{D}$, the energy conservation reads

$$
\frac{d}{d t} \mathcal{E}-\int_{\partial \mathcal{D}} d S \boldsymbol{\Pi} \cdot \mathbf{n}=0
$$

with $\mathcal{E}$ the elastic energy and $\boldsymbol{\Pi}$ the Poynting vector, or elastic energy flux,

$$
\mathcal{E}=\int_{\mathcal{D}} d V\left[\frac{\rho}{2} V^{2}+\frac{1}{2 \mu} \boldsymbol{\Sigma}^{2}\right], \quad \boldsymbol{\Pi}=V \boldsymbol{\Sigma} .
$$

We consider wave sources imposing in the elastic matrix a minimum wavelength $2 \pi / k$ larger than the periodicity of the array $h$, such that

$$
\eta \equiv k h \ll 1
$$

and the array thickness $e=O(h)$. Next, to be consistent, we shall work in dimensionless coordinates, and we define

$$
\mathbf{x} \equiv k \mathbf{X}, \quad \tau \equiv k \sqrt{\frac{\mu_{\mathrm{m}}}{\rho_{\mathrm{m}}}} t,
$$


and

$$
\boldsymbol{\Sigma}(\mathbf{X}, t) \equiv \sqrt{\rho_{\mathrm{m}} \mu_{\mathrm{m}}} \boldsymbol{\sigma}(\mathbf{x}, \tau), \quad V(\mathbf{X}, t) \equiv v(\mathbf{x}, \tau),
$$

where $\left(\rho_{\mathrm{m}}, \mu_{\mathrm{m}}\right)$ are the mass density and the shear modulus of the elastic matrix, and we denote $\left(\rho_{\mathrm{i}}, \mu_{\mathrm{i}}\right)$ those of the inclusions. Now, the Eqs. 22 read in dimensionless form

$$
\left\{\begin{array}{c}
\frac{\partial \boldsymbol{\sigma}}{\partial \tau}=\frac{\mu}{\mu_{\mathrm{m}}} \nabla_{\mathbf{x}} v \\
\frac{\rho}{\rho_{\mathrm{m}}} \frac{\partial v}{\partial \tau}=\operatorname{div}_{\mathbf{x}} \boldsymbol{\sigma},
\end{array}\right.
$$

with $\mathbf{x} \in \mathbb{R}^{2}$. At the boundaries between the matrix and the inclusions, the continuities of the displacement, and thus of its time derivative $v$, and the continuity of the normal stress $\sigma . \mathbf{n}$ apply; finally, appropriate boundary condition at $|\mathbf{x}| \rightarrow+\infty$, often referred to as radiation conditions, apply once the wave source has been defined; they are in general of the Sommerfeld type, and consist in selecting the waves propagating away from the sources (by opposition to their time reversed counterparts which propagate from infinity toward the sources). For the time being, we do not need to specify their form.

\subsection{The inner and outer expansions}

The solutions of Eqs. (3) can be expanded with respect to the small parameter $\eta$, namely

$$
\left\{\begin{array}{l}
v=v^{0}(\mathbf{x}, \tau)+\eta v^{1}(\mathbf{x}, \tau)+\eta^{2} v^{2}(\mathbf{x}, \tau)+\ldots \\
\boldsymbol{\sigma}=\boldsymbol{\sigma}^{0}(\mathbf{x}, \tau)+\eta \boldsymbol{\sigma}^{1}(\mathbf{x}, \tau)+\eta^{2} \boldsymbol{\sigma}^{2}(\mathbf{x}, \tau)+\ldots
\end{array}\right.
$$

While in principle the above expansion could be used in the whole space, we consider a separation of the space into an inner region $\left(\left|x_{1}\right| \ll 1\right)$ and an outer region $\left(\left|x_{1}\right| \gg \eta\right)$, which correspond in terms of wavefield to the near field and to the far field, respectively. In the outer region, the natural coordinate $\mathbf{x} \equiv\left(x_{1}, x_{2}\right)$ is adapted to describe the slow variations (in $\left.1 / k\right)$ of the wavefield, and the expansions in Eq. (4) are used. In the inner region, a new system of coordinates $\mathbf{y}=\mathbf{x} / \eta$ is used which accounts for the rapid variations (in $h$ ) of the evanescent fields. Next, the slow variations along $x_{2}$ are accounted for by keeping $x_{2}$ as additional coordinate. Owing to these considerations, the expansions read

$$
\begin{aligned}
& \text { Outer exp. }\left\{\begin{array}{l}
v=v^{0}(\mathbf{x}, \tau)+\eta v^{1}(\mathbf{x}, \tau)+\ldots, \\
\boldsymbol{\sigma}=\boldsymbol{\sigma}^{0}(\mathbf{x}, \tau)+\eta \boldsymbol{\sigma}^{1}(\mathbf{x}, \tau)+\ldots,
\end{array}\right. \\
& \text { Inner exp. }\left\{\begin{array}{l}
v=w^{0}\left(\mathbf{y}, x_{2}, \tau\right)+\eta w^{1}\left(\mathbf{y}, x_{2}, \tau\right)+\ldots, \\
\boldsymbol{\sigma}=\boldsymbol{\tau}^{0}\left(\mathbf{y}, x_{2}, \tau\right)+\eta \boldsymbol{\tau}^{1}\left(\mathbf{y}, x_{2}, \tau\right)+\ldots,
\end{array}\right.
\end{aligned}
$$


and the terms $\left(w^{n}, \boldsymbol{\tau}^{n}\right), n=0,1, \ldots$, of the inner solution are assumed to be periodic w.r.t. $y_{2}$. Thus, we shall consider in the outer region $\mathbf{x} \in \mathbb{R}^{2}$, and in the inner region $\mathbf{y} \in \mathbb{R} \times Y$, with $Y=(-1 / 2,1 / 2)$ (Fig. 2).

Now, Eqs. (3) can be written in the inner and in the outer regions, owing to the expressions of the differential operator

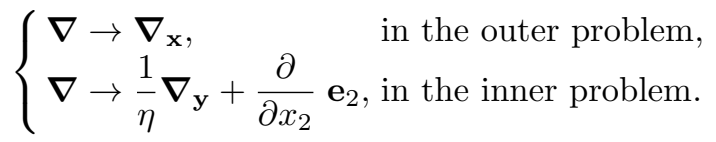

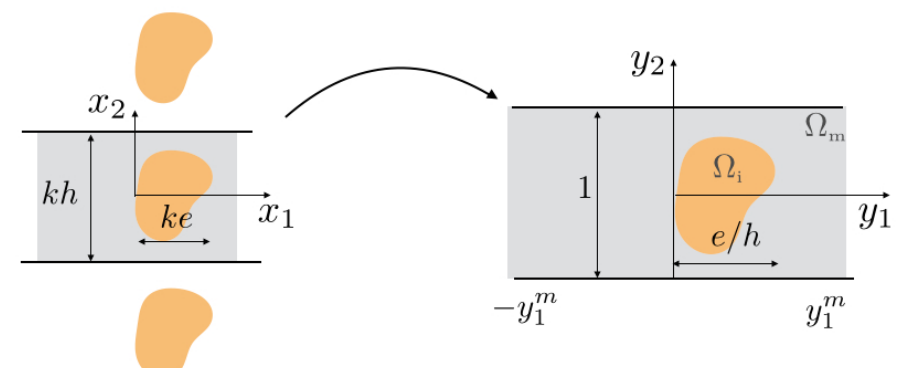

Figure 2 The array of inclusions in $\mathbf{x}$-coordinates, with $\mathbf{x} \in \mathbb{R}^{2}$, and the elementary cell in $\mathbf{y}$-coordinates with $\mathbf{y} \in \mathbb{R} \times Y(Y=(-1 / 2,1 / 2))$. In $\mathbf{y}$-coordinates, we define $\Omega=$ $\left(-y_{1}^{m}, y_{1}^{m}\right) \times Y$, with $y_{1}^{m}>e / h$ (in practice $y_{1}^{m} \rightarrow+\infty$ will be considered); $\Omega=\Omega_{\mathrm{i}} \cup \Omega_{\mathrm{m}}$, with $\Omega_{\mathrm{i}}$ and $\Omega_{\mathrm{m}}$ the domains occupied by the inclusion and by the matrix, respectively.

In the following, we shall use the domain $\Omega=\left(-y_{1}^{m}, y_{1}^{m}\right) \times Y$ in $\mathbf{y}$ coordinate containing a single inclusion (Fig. 2). $\Omega_{\mathrm{i}}$ and $\Omega_{\mathrm{m}}$ are the subdomains occupied by the inclusion and by the matrix respectively $\left(\Omega=\Omega_{\mathrm{i}} \cup \Omega_{\mathrm{m}}\right)$; the continuities of the displacement and of the normal stress apply on $\partial \Omega_{\mathrm{i}}$. We shall use also $\Omega_{\infty}=\lim _{y_{1}^{m} \rightarrow+\infty} \Omega$.

\subsection{Boundary conditions and matching conditions}

Because of the separation of the space into two regions, something has to be said on the boundary conditions. By construction, the continuity relations at the boundary of the inclusions apply for the inner solution only, whence

$$
w^{n} \text { and } \boldsymbol{\tau}^{n} \cdot \mathbf{n} \text { are continuous on } \partial \Omega_{\mathrm{i}}, \text { for } n=0,1, \ldots
$$

but boundary conditions at $\left|y_{1}\right| \rightarrow+\infty$ are missing. Reversely, the outer solution is submitted to the radiation conditions at $|\mathbf{x}| \rightarrow+\infty$ but the boundary conditions for $x_{1} \rightarrow 0^{ \pm}$are unknown a priori, and it is in fact the boundary conditions that will provide the jump conditions. These missing conditions for the inner and outer terms are given simultanesouly by so-called matching 
conditions, which tell us that the two solutions have to match in some intermediate region. Following [7] the matching is written for $x_{1} \rightarrow 0^{ \pm}$corresponding to $y_{1} \rightarrow \pm \infty$ (and we denote $f\left(0^{ \pm}\right)$the limit values of $f$ for $x_{1} \rightarrow 0^{ \pm}$). To do so, we use the Taylor expansions of $v^{0}\left(x_{1}, x_{2}, \tau\right)=v^{0}\left(0^{ \pm}, x_{2}, \tau\right)+$ $x_{1} \partial_{x_{1}} v^{0}\left(0^{ \pm}, x_{2}, \tau\right)+\cdots=v^{0}\left(0^{ \pm}, x_{2}, \tau\right)+\eta y_{1} \partial_{x_{1}} v^{0}\left(0^{ \pm}, x_{2}, \tau\right)+\ldots$, same for

$\boldsymbol{\sigma}^{0}$. Identifying the terms in $\eta^{n}, n=0,1$ in the inner and outer expansions, Eqs. (5), we get, for $n=0$

$$
\left\{\begin{array}{l}
v^{0}\left(0^{ \pm}, x_{2}, \tau\right)=\lim _{y_{1} \rightarrow+\infty} w^{0}\left(\mathbf{y}, x_{2}, \tau\right), \\
\boldsymbol{\sigma}^{0}\left(0^{ \pm}, x_{2}, \tau\right)=\lim _{y_{1} \rightarrow+\infty} \boldsymbol{\tau}^{0}\left(\mathbf{y}, x_{2}, \tau\right),
\end{array}\right.
$$

and for $n=1$,

$$
\left\{\begin{array}{l}
v^{1}\left(0^{ \pm}, x_{2}, \tau\right)=\lim _{y_{1} \rightarrow+\infty}\left[w^{1}\left(\mathbf{y}, x_{2}, \tau\right)-y_{1} \frac{\partial v^{0}}{\partial x_{1}}\left(0, x_{2}, \tau\right)\right], \\
\boldsymbol{\sigma}^{1}\left(0 \pm, x_{2}, \tau\right)=\lim _{y_{1} \rightarrow+\infty}\left[\boldsymbol{\tau}^{1}\left(\mathbf{y}, x_{2}, \tau\right)-y_{1} \frac{\partial \boldsymbol{\sigma}^{0}}{\partial x_{1}}\left(0, x_{2}, \tau\right)\right] .
\end{array}\right.
$$

When not needed, the dependance of the functions on the spatial variables and on the time will be omitted for readability.

\subsection{Jump conditions at the first order}

We start with the jump conditions at the first order $\llbracket v^{0} \rrbracket$ and $\llbracket \sigma_{1}^{0} \rrbracket$, where we defined for any outer terms $f$

$$
\llbracket f \rrbracket \equiv f\left(0^{+}, x_{2}, \tau\right)-f\left(0^{-}, x_{2}, \tau\right) .
$$

Eqs. (3) at the leading order in $\eta^{-1}$ give

$$
\nabla_{\mathbf{y}} w^{0}=0, \quad \operatorname{div}_{\mathbf{y}} \boldsymbol{\tau}^{0}=0,
$$

from which we deduce that

$$
v^{0}\left(0, x_{2}, \tau\right)=v^{0}\left(0^{ \pm}, x_{2}, \tau\right)=w^{0}\left(x_{2}\right) .
$$

Next, integrating $\operatorname{div}_{\mathbf{y}} \boldsymbol{\tau}^{0}=0$ over $\Omega$, and using (i) the continuity of $\boldsymbol{\tau}^{0} \cdot \mathbf{n}$ on $\partial \Omega_{i}$, Eq. (6), and (ii) the periodicity of $\boldsymbol{\tau}^{0}$, we get

$$
\int_{Y} \mathrm{~d} y_{2}\left[\tau_{1}^{0}\left(+y_{1}^{m}, y_{2}, x_{2}, \tau\right)-\tau_{1}^{0}\left(-y_{1}^{m}, y_{2}, x_{2}, \tau\right)\right]=0 .
$$

Finally, taking the limit $y_{1}^{m} \rightarrow+\infty$ in the above equation and integrating the matching condition $7 \mathrm{~b}$ over $Y$, we get

$$
\int_{Y} \mathrm{~d} y_{2} \tau_{1}^{0}\left( \pm \infty, y_{2}, x_{2}, \tau\right)=\sigma_{1}^{0}\left(0^{ \pm}, x_{2}, \tau\right) .
$$


We deduce from (11)- 12 the jump conditions at the first order

$$
\llbracket u^{0} \rrbracket=\llbracket \sigma_{1}^{0} \rrbracket=0 .
$$

2.4 Elementary problems and jump of $u^{1}$

To get the jumps at second order, we need to define the problem satisfied by $\left(w^{1}, \boldsymbol{\tau}^{0}\right)$. To do that, we use the time derivative of 10

$$
\operatorname{div}_{\mathbf{y}} \frac{\partial \tau^{0}}{\partial \tau}=0
$$

Eq. (3a) written at the order $\eta^{0}$ for the inner solution, along with (11), gives

$$
\frac{\partial \boldsymbol{\tau}^{0}}{\partial \tau}=\frac{\mu(\mathbf{y})}{\mu_{\mathrm{m}}}\left(\nabla_{\mathbf{y}} w^{1}+\frac{\partial v^{0}}{\partial x_{2}}\left(0, x_{2}, \tau\right) \mathbf{e}_{2}\right)
$$

It follows that the system satisfied by $w^{1}$ reads

$$
\left\{\begin{array}{l}
\operatorname{div}_{\mathbf{y}}\left[\mu(\mathbf{y})\left(\boldsymbol{\nabla}_{\mathbf{y}} w^{1}+\frac{\partial v^{0}}{\partial x_{2}}\left(0, x_{2}, \tau\right)\right)\right]=0 \\
w^{1} \text { and } \mu(\mathbf{y})\left(\boldsymbol{\nabla}_{\mathbf{y}} w^{1}+\frac{\partial v^{0}}{\partial x_{2}}\left(0, x_{2}, \tau\right)\right) \cdot \mathbf{n} \text { continuous on } \partial \Omega_{\mathrm{i}}, \\
\lim _{y_{1} \rightarrow \pm \infty} \boldsymbol{\nabla}_{\mathbf{y}} w^{1}=\frac{\partial v^{0}}{\partial x_{1}}\left(0, x_{2}, \tau\right) \mathbf{e}_{1} .
\end{array}\right.
$$

The first equation in the bulk uses simply (14)-(15). The continuity relations on $\partial \Omega_{\mathrm{i}}$ come from (6) (for $\boldsymbol{\tau}^{0} . \mathbf{n}$, it is the time derivative version of the continuity relation). The third equation is less immediate although straightforward ; consider the time derivative of Eq. (7b), with Eq. 15)

$$
\frac{\partial \boldsymbol{\sigma}^{0}}{\partial \tau}\left(0^{ \pm}, x_{2}, \tau\right)=\lim _{y_{1} \rightarrow \pm \infty} \nabla_{\mathbf{y}} w^{1}+\frac{\partial v^{0}}{\partial x_{2}}\left(0, x_{2}, \tau\right) \mathbf{e}_{2},
$$

where we used that $\mu(\mathbf{y}) \rightarrow \mu_{\mathrm{m}}$ for $y_{1} \rightarrow \pm \infty$. It is now sufficient to use (3a) at the order $\eta^{0}$ for the outer solution, namely

$$
\frac{\partial \boldsymbol{\sigma}^{0}}{\partial \tau}=\nabla_{\mathbf{x}} v^{0}
$$

which leads to the desired (third) equation in (16). The system 16 is linear with respect to $\partial_{x_{1}} v^{0}\left(0, x_{2}, \tau\right)$ and $\partial_{x_{2}} v^{0}\left(0, x_{2}, \tau\right)$. Thus, to ensure that $w^{1}$ satisfies 16 , it is sufficient to define $W^{(1)}$ and $W^{(2)}$ such as

$w^{1}\left(\mathbf{y}, x_{2}, \tau\right)=\frac{\partial v^{0}}{\partial x_{1}}\left(0, x_{2}, \tau\right)\left[W^{(1)}(\mathbf{y})+y_{1}\right]+\frac{\partial v^{0}}{\partial x_{2}}\left(0, x_{2}, \tau\right) W^{(2)}(\mathbf{y})+W\left(x_{2}, \tau\right)$, 
with $W^{(\mathrm{i})}, i=1,2$, satisfying

$$
\left\{\begin{array}{l}
\operatorname{div}\left[\mu \boldsymbol{\nabla}\left(W^{(\mathrm{i})}+y_{i}\right)\right]=0, \\
W^{(\mathrm{i})} \text { and } \mu \boldsymbol{\nabla}\left(W^{(\mathrm{i})}+y_{i}\right) \cdot \mathbf{n} \text { are continuous on } \partial \Omega_{\mathrm{i}}, \\
\lim _{y_{1} \rightarrow \pm \infty} \boldsymbol{\nabla} W^{(\mathrm{i})}=\mathbf{0},
\end{array}\right.
$$

The field $W^{(1)}$ and $W^{(2)}$ at $y_{1} \rightarrow \pm \infty$ are evanescent fields up to a constant, being possibly different at the two limits. In the following, we define $\mathcal{B}_{i}, i=1,2$, as

$$
\mathcal{B}_{i} \equiv W^{(\mathrm{i})}\left(+\infty, y_{2}\right)-W^{(\mathrm{i})}\left(-\infty, y_{2}\right) .
$$

Finally, note that $W\left(x_{2}, \tau\right)$ has been introduced in (17) since the Eqs. (16) define $w^{1}$ up to a function independent of $\mathbf{y}$; we shall see that the determination of $W$ is not needed. The jump of $v^{1}$ is obtained from the matching condition (8a), with 17) and (19), namely

$$
\llbracket v^{1} \rrbracket=\mathcal{B}_{1} \frac{\partial v^{0}}{\partial x_{1}}\left(0, x_{2}, \tau\right)+\mathcal{B}_{2} \frac{\partial v^{0}}{\partial x_{2}}\left(0, x_{2}, \tau\right) .
$$

Note that $\partial_{x_{1}} v^{0}$ is continuous at $x_{1}=0$, since $\partial_{\tau} \sigma_{1}^{0}=\partial_{x_{1}} v^{0}$, from (3a), and $\sigma_{1}^{0}$ is continuous; obviously, $\partial_{x_{2}} v^{0}$ is continuous also, with $v^{0}\left(0, x_{2}, \tau\right)$ being continuous.

\subsubsection{Jump of $\sigma_{1}^{1}$}

The derivation of the jump condition on $\sigma_{1}^{1}$ is less straightforward. We shall integrate (3b) at order $\eta^{0}$ for the inner solution over $\Omega$, specifically

$$
\frac{\rho(\mathbf{y})}{\rho_{\mathrm{m}}} \frac{\partial v^{0}}{\partial \tau}\left(0, x_{2}, \tau\right)=\operatorname{div}_{\mathbf{y}} \tau^{1}+\frac{\partial \tau_{2}^{0}}{\partial x_{2}},
$$

where we used (11). Before doing so, we inspect the term $\tau_{2}^{0}$ which is needed in the equation above. From (15) and (17), we have

$$
\frac{\partial \tau_{2}^{0}}{\partial \tau}=\frac{\mu(\mathbf{y})}{\mu_{\mathrm{m}}}\left[\frac{\partial v^{0}}{\partial x_{1}}\left(0, x_{2}, \tau\right) \frac{\partial W^{(1)}}{\partial y_{2}}+\frac{\partial v^{0}}{\partial x_{2}}\left(0, x_{2}, \tau\right)\left(\frac{\partial W^{(2)}}{\partial y_{2}}+1\right)\right] .
$$

It is now sufficient to use $3 \mathrm{a}$ for the outer problem at the order $\eta^{0}$ to get

$$
\frac{\partial \sigma_{1}^{0}}{\partial \tau}=\frac{\partial v^{0}}{\partial x_{1}}, \quad \frac{\partial \sigma_{2}^{0}}{\partial \tau}=\frac{\partial v^{0}}{\partial x_{2}}
$$

and thus

$$
\tau_{2}^{0}=\frac{\mu(\mathbf{y})}{\mu_{\mathrm{m}}}\left[\sigma_{1}^{0}\left(0, x_{2}, \tau\right) \frac{\partial W^{(1)}}{\partial y_{2}}+\sigma_{2}^{0}\left(0, x_{2}, \tau\right)\left(\frac{\partial W^{(2)}}{\partial y_{2}}+1\right)\right] .
$$

We have assumed that $\sigma^{0}=0$ and $\tau^{0}=0$ at $\tau=-\infty$ (the source has been switched on at some initial time), so that the above identity has been written 
omitting the time derivative. We can come back to the Eq. 21], integrated over $\Omega$,

$$
\frac{\partial v^{0}}{\partial \tau}\left(0, x_{2}, \tau\right) \int_{\Omega} \mathrm{d} \mathbf{y} \frac{\rho(\mathbf{y})}{\rho_{\mathrm{m}}}-\int_{\Omega} \mathrm{d} \mathbf{y}\left[\operatorname{div}_{\mathbf{y}} \boldsymbol{\tau}^{1}+\frac{\partial \tau_{2}^{0}}{\partial x_{2}}\right]=0 .
$$

Eq. (23) involve three integrals. The first integral is

$$
\frac{\partial v^{0}}{\partial \tau}\left(0, x_{2}, \tau\right) \int_{\Omega} \mathrm{d} \mathbf{y} \frac{\rho(\mathbf{y})}{\rho_{\mathrm{m}}}=\frac{\partial v^{0}}{\partial \tau}\left(0, x_{2}, \tau\right)\left[2 y_{1}^{m}+\frac{e \varphi}{h}\left(\frac{\rho_{\mathrm{i}}}{\rho_{\mathrm{m}}}-1\right)\right],
$$

with $e \varphi / h$ the surface of the inclusion in $\mathbf{y}$-coordinate $(\varphi$ is the filling fraction of inclusion for $y_{1} \in(0, e / h)$, see Fig. 2). The second integral, of $\operatorname{div}_{\mathbf{y}} \tau^{1}$, is obtained owing to the continuity of $\tau^{1} . \mathbf{n}$ and its periodicity w.r.t. $y_{2}$, and we get

$$
-\int_{\Omega} \mathrm{d} \mathbf{y} \operatorname{div}_{\mathbf{y}} \boldsymbol{\tau}_{1}=-\int_{Y} \mathrm{~d} y_{2}\left[\tau_{1}^{1}\left(+y_{1}^{m}, y_{2}, x_{2}, \tau\right)-\tau_{1}^{1}\left(-y_{1}^{m}, y_{2}, x_{2}, \tau\right)\right] .
$$

For the third integral, with $\partial \tau_{2}^{0} / \partial x_{2}$ given by 22 , we have

$$
\begin{aligned}
-\int_{\Omega} \mathrm{d} \mathbf{y} \frac{\partial \tau_{2}^{0}}{\partial x_{2}}= & -\frac{\partial \sigma_{1}^{0}}{\partial x_{2}}\left(0, x_{2}, \tau\right) \int_{\Omega} \frac{\mu(\mathbf{y})}{\mu_{\mathrm{m}}} \frac{\partial W^{(1)}}{\partial y_{2}} \\
& -\frac{\partial \sigma_{2}^{0}}{\partial x_{2}}\left(0, x_{2}, \tau\right)\left[\int_{\Omega} \frac{\mu(\mathbf{y})}{\mu_{\mathrm{m}}} \frac{\partial W^{(2)}}{\partial y_{2}}+2 y_{1}^{m}+\frac{e \varphi}{h}\left(\frac{\mu_{\mathrm{i}}}{\mu_{\mathrm{m}}}-1\right)\right] .
\end{aligned}
$$

Two terms, in (24) and in (26), are linear in $y_{1}^{m}$. Let us sum them

$$
2 y_{1}^{m}\left[\frac{\partial v^{0}}{\partial \tau}\left(0, x_{2}, \tau\right)-\frac{\partial \sigma_{2}^{0}}{\partial x_{2}}\left(0, x_{2}, \tau\right)\right]=2 y_{1}^{m} \frac{\partial \sigma_{1}^{0}}{\partial x_{1}}\left(0, x_{2}, \tau\right),
$$

where we have used $3 \mathrm{~b}$ for the inner problem at order $\eta^{0}$, namely

$$
\frac{\partial \sigma_{1}^{0}}{\partial x_{1}}+\frac{\partial \sigma_{2}^{0}}{\partial x_{2}}=\frac{\partial v^{0}}{\partial \tau} .
$$

Now, we gather the two terms in 25) and 27) using the matching conditions (8b) written in a different form

$$
\left\{\begin{array}{l}
\sigma_{1}^{1}\left(0^{-}, x_{2}, \tau\right)=\lim _{y_{1}^{m} \rightarrow+\infty}\left[\tau_{1}^{1}\left(-y_{1}^{m}, y_{2}, x_{2}, \tau\right)+y_{1}^{m} \frac{\partial \sigma_{1}^{0}}{\partial x_{1}}\left(0^{-}, x_{2}, \tau\right)\right] \\
\sigma_{1}^{1}\left(0^{+}, x_{2}, \tau\right)=\lim _{y_{1}^{m} \rightarrow+\infty}\left[\tau_{1}^{1}\left(y_{1}^{m}, y_{2}, x_{2}, \tau\right)-y_{1}^{m} \frac{\partial \sigma_{1}^{0}}{\partial x_{1}}\left(0^{+}, x_{2}, \tau\right)\right]
\end{array}\right.
$$

and owing to the continuity of $\sigma_{1}^{0}$ at $y_{1}=0$, we get

$$
\llbracket \sigma_{1}^{1} \rrbracket=\lim _{y_{1}^{m} \rightarrow \infty}\left[\tau_{1}^{1}\left(y_{1}^{m}, y_{2}, x_{2}, \tau\right)-\tau_{1}^{1}\left(-y_{1}^{m}, y_{2}, x_{2}, \tau\right)-2 y_{1}^{m} \frac{\partial \sigma_{1}^{0}}{\partial x_{1}}\left(0, x_{2}, \tau\right)\right] \text {. }
$$


Collecting Eqs. 24) to (27) in 23) for $y_{1}^{m} \rightarrow+\infty$, and using (30), we get

$$
\begin{aligned}
\llbracket \sigma_{1}^{1} \rrbracket= & \frac{\partial v^{0}}{\partial \tau}\left(0, x_{2}, \tau\right) \frac{e \varphi}{h}\left(\frac{\rho_{\mathrm{i}}}{\rho_{\mathrm{m}}}-1\right)-\frac{\partial \sigma_{1}^{0}}{\partial x_{2}}\left(0, x_{2}, \tau\right) \int_{\Omega_{\infty}} \frac{\mu(\mathbf{y})}{\mu_{\mathrm{m}}} \frac{\partial W^{(1)}}{\partial y_{2}} \\
& -\frac{\partial \sigma_{2}^{0}}{\partial x_{2}}\left(0, x_{2}, \tau\right)\left[\int_{\Omega_{\infty}} \frac{\mu(\mathbf{y})}{\mu_{\mathrm{m}}} \frac{\partial W^{(2)}}{\partial y_{2}}+\frac{e \varphi}{h}\left(\frac{\mu_{\mathrm{i}}}{\mu_{\mathrm{m}}}-1\right)\right] .
\end{aligned}
$$

Finally, with 28), the jump of $\sigma_{1}^{1}$ reads

$$
\begin{aligned}
\llbracket \sigma_{1}^{1} \rrbracket= & \frac{e \varphi}{h}\left(\frac{\rho_{\mathrm{i}}}{\rho_{\mathrm{m}}}-1\right) \frac{\partial \sigma_{1}^{0}}{\partial x_{1}}\left(0, x_{2}, \tau\right)-\mathcal{C}_{1} \frac{\partial \sigma_{1}^{0}}{\partial x_{2}}\left(0, x_{2}, \tau\right) \\
& -\left[\mathcal{C}_{2}+\frac{e \varphi}{h}\left(\frac{\mu_{\mathrm{i}}}{\mu_{\mathrm{m}}}-\frac{\rho_{\mathrm{i}}}{\rho_{\mathrm{m}}}\right)\right] \frac{\partial \sigma_{2}^{0}}{\partial x_{2}}\left(0, x_{2}, \tau\right),
\end{aligned}
$$

where we have defined, for $i=1,2$,

$$
\mathcal{C}_{i} \equiv \int_{\Omega_{\infty}} \mathrm{d} \mathbf{y} \frac{\mu(\mathbf{y})}{\mu_{\mathrm{m}}} \frac{\partial W^{(\mathrm{i})}}{\partial y_{2}} .
$$

\subsection{Final jump conditions}

It has already been stressed in [1,2,5,6] that the jump conditions can be expressed in a different form, equivalent up to $O\left(\eta^{2}\right)$ to those obtained for $v^{0}+\eta v^{1}$ and for $\sigma_{1}^{0}+\eta \sigma_{1}^{1}$. This form relies on the following consideration. With $e$ the thickness of the inclusions, the array in $\mathbf{y}$-coordinates has a rescaled thickness $k e=\eta e / h$ in $\mathbf{x}$-coordinate. We consider $a>0$ the size of the enlarged interface in the real space, and we assume that $a / h=O(1)$. Thus, for any $f=v$ or $f=\sigma_{1}$, we have

$$
f\left(k a, x_{2}, \tau\right)=f\left(0^{+}, x_{2}, \tau\right)+\eta \frac{a}{h} \frac{\partial f}{\partial x_{1}}\left(k a, x_{2}, \tau\right)+O\left(\eta^{2}\right),
$$

from which, defining $\llbracket f \rrbracket_{a} \equiv f(a)-f\left(0^{-}\right)$for the $a$-enlarged interface, we have

$$
\llbracket f \rrbracket_{a}=\llbracket f \rrbracket+\eta \frac{a}{h} \frac{\partial f}{\partial x_{1}}\left(k a, x_{2}, \tau\right)+O\left(\eta^{2}\right) .
$$

With $f=f^{0}+\eta f^{1}+O\left(\eta^{2}\right)$, (35) gives

$$
\llbracket f \rrbracket_{a}=\llbracket f^{0} \rrbracket_{a}+\eta \llbracket f^{1} \rrbracket_{a}+O\left(\eta^{2}\right)=\eta\left[\frac{a}{h} \frac{\partial f^{0}}{\partial x_{1}}\left(k a, x_{2}, \tau\right)+\llbracket f^{1} \rrbracket\right]+O\left(\eta^{2}\right),
$$

where we have used that $\llbracket f^{0} \rrbracket=0$ and that $\llbracket f^{1} \rrbracket_{a}=\llbracket f^{1} \rrbracket+O(\eta)$. We shall use also that, with

$$
\langle f\rangle \equiv \frac{1}{2}\left[f\left(0^{-}\right)+f\left(0^{+}\right)\right], \quad\langle f\rangle_{a} \equiv \frac{1}{2}\left[f\left(0^{-}\right)+f(k a)\right],
$$

we have

$$
\langle f\rangle_{a}=\left\langle f^{0}\right\rangle_{a}+O(\eta)=f^{0}\left(0, x_{2}, \tau\right)+O(\eta),
$$


since $f^{0}$ is continuous at $x_{1}=0$. We use $(36)$ and $(38)$ in the jump conditions (20) and (32) to finally get

$$
\left\{\begin{array}{l}
\llbracket v \rrbracket_{a}=\eta \mathcal{B}\left\langle\frac{\partial v}{\partial x_{1}}\right\rangle_{a}+\eta \mathcal{B}_{2}\left\langle\frac{\partial v}{\partial x_{2}}\right\rangle_{a}+O\left(\eta^{2}\right), \\
\llbracket \sigma_{1} \rrbracket_{a}=\eta\left[\mathcal{S}\langle\operatorname{div} \boldsymbol{\sigma}\rangle_{a}-\mathcal{C}_{1}\left\langle\frac{\partial \sigma_{1}}{\partial x_{2}}\right\rangle_{a}-\mathcal{C}\left\langle\frac{\partial \sigma_{2}}{\partial x_{2}}\right\rangle_{a}\right]_{a}+O\left(\eta^{2}\right),
\end{array}\right.
$$

with

$$
\mathcal{B} \equiv \frac{a}{h}+\mathcal{B}_{1}, \mathcal{C} \equiv \frac{a}{h}+\frac{e \varphi}{h}\left(\frac{\mu_{\mathrm{i}}}{\mu_{\mathrm{m}}}-1\right)+\mathcal{C}_{2}, \mathcal{S} \equiv \frac{a}{h}+\frac{e \varphi}{h}\left(\frac{\rho_{\mathrm{i}}}{\rho_{\mathrm{m}}}-1\right) .
$$

Following [8], we define the final jump condition for the field $\left(v^{h}, \boldsymbol{\sigma}^{h}\right)$ satisfying the Helmholtz equation in the matrix and the jump conditions

$$
\left\{\begin{array}{l}
\llbracket v^{h} \rrbracket_{a}=\eta \mathcal{B}\left\langle\frac{\partial v^{h}}{\partial x_{1}}\right\rangle_{a}+\eta \mathcal{B}_{2}\left\langle\frac{\partial v^{h}}{\partial x_{2}}\right\rangle_{a} \\
\llbracket \sigma_{1}^{h} \rrbracket_{a}=\eta\left[\mathcal{S}\left\langle\operatorname{div} \boldsymbol{\sigma}^{h}\right\rangle_{a}-\mathcal{C}_{1}\left\langle\frac{\partial \sigma_{1}^{h}}{\partial x_{2}}\right\rangle_{a}-\mathcal{C}\left\langle\frac{\partial \sigma_{2}^{h}}{\partial x_{2}}\right\rangle_{a}\right]_{a}
\end{array}\right.
$$

and it is easy to see that $\left(v^{h}, \boldsymbol{\sigma}^{h}\right)$ have the same expansions as $(v, \boldsymbol{\sigma})$ up to $O\left(\eta^{2}\right)$. Coming back to the real space in $\mathbf{X}$ - coordinate, we get the final jump conditions at the second order

$$
\left\{\begin{array}{l}
\llbracket V \rrbracket_{a}=h \mathcal{B}\left\langle\frac{\partial V}{\partial X_{1}}\right\rangle_{a}+h \mathcal{B}_{2}\left\langle\frac{\partial V}{\partial X_{2}}\right\rangle_{a}, \\
\llbracket \Sigma_{1} \rrbracket_{a}=\left[h \mathcal{S}\langle\operatorname{div} \boldsymbol{\Sigma}\rangle_{a}-h \mathcal{C}_{1}\left\langle\frac{\partial \Sigma_{1}}{\partial X_{2}}\right\rangle_{a}-h \mathcal{C}\left\langle\frac{\partial \Sigma_{2}}{\partial X_{2}}\right\rangle_{a}\right] .
\end{array}\right.
$$

2.6 Energy conservation in the homogenized problem

Below, we address the equation of energy conservation. In the original problem, the elastic energy is

$$
\mathcal{E}=\int_{\mathcal{D}} d V\left[\frac{\rho}{2} V^{2}+\frac{1}{2 \mu} \Sigma^{2}\right]
$$

and in the homogenized problem, it is

$$
\mathcal{E}_{h}=\int_{\mathcal{D} \backslash \mathcal{D}_{a}} d V\left[\frac{\rho}{2} V^{2}+\frac{1}{2 \mu} \Sigma^{2}\right]+\mathcal{E}_{a},
$$

where $\mathcal{E}_{a}$ is the energy of the equivalent interface of thickness $a$, that we expect to account for the elastic energy in the original interface of thickness $e$ containing the inclusions, thus that we expect to be positive (Fig. 3). Let 


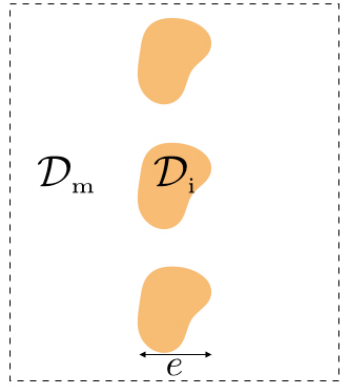

(a)

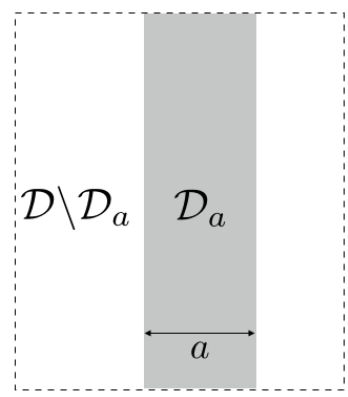

(b)

Figure 3 (a) In the real problem, the elastic energy is defined in $\mathcal{D}=\mathcal{D}_{\mathrm{i}} \cup \mathcal{D}_{\mathrm{m}}$, (b) in the homogenized problem, the energy is defined in $\mathcal{D} \backslash \mathcal{D}_{a}$; an additional term arises from the jump conditions between the boundaries of the equivalent interface $\mathcal{D}_{a}$ of thickness $a$.

us inspect the expression of $\mathcal{E}_{a}$. The energy conservation in the homogenized problem reads

$$
\frac{d}{d t} \int_{\mathcal{D} \backslash \mathcal{D}_{a}} d V\left[\frac{\rho}{2} V^{2}+\frac{1}{2 \mu} \boldsymbol{\Sigma}^{2}\right]-\int_{\partial\left(\mathcal{D} \backslash \mathcal{D}_{a}\right)} d S \boldsymbol{\Pi} . \mathbf{n}=0 .
$$

The fluxes of the Poynting vector $-\int_{\partial\left(\mathcal{D} \backslash \mathcal{D}_{a}\right)} d S \boldsymbol{\Pi} . \mathbf{n}$ (with $\boldsymbol{\Pi}=V \boldsymbol{\Sigma}$ ) involve notably $I \equiv \int \mathrm{d} X_{2} \llbracket V \Sigma_{1} \rrbracket_{a}$ inside the domain; the change in the sign is due to the convention of outer pointing normal vector $\mathbf{n}$ for $\partial\left(\mathcal{D} \backslash \mathcal{D}_{a}\right)$.

We shall see that $I$ is the time derivative of an energy supported by the interface. Applying the jump conditions, Eqs. 42, we get

$$
\begin{aligned}
I= & \int \mathrm{d} X_{2} h\left[\mathcal{B}\left\langle\frac{\partial V}{\partial X_{1}}\right\rangle_{a}+\mathcal{B}_{2}\left\langle\frac{\partial V}{\partial X_{2}}\right\rangle_{a}\right]\left\langle\Sigma_{1}\right\rangle_{a} \\
& +\int \mathrm{d} X_{2} h\left[\mathcal{S}\langle\operatorname{div} \Sigma\rangle_{a}-\mathcal{C}_{1}\left\langle\frac{\partial \Sigma_{1}}{\partial X_{2}}\right\rangle_{a}-\mathcal{C}\left\langle\frac{\partial \Sigma_{2}}{\partial X_{2}}\right\rangle_{a}\right]\langle V\rangle_{a},
\end{aligned}
$$

and using Eqs. (2) (with $\mu=\mu_{\mathrm{m}}$ and $\rho=\rho_{\mathrm{m}}$ ),

$$
I=\frac{d}{d t} \mathcal{E}_{a}+h\left(\mathcal{B}_{2}+\mathcal{C}_{1}\right) \int \mathrm{d} X_{2}\left\langle\frac{\partial V}{\partial X_{2}}\right\rangle_{a}\left\langle\Sigma_{1}\right\rangle_{a}=\frac{d}{d t} \mathcal{E}_{a}
$$

with

$$
\mathcal{E}_{a}=\int \mathrm{d} X_{2} h\left[\frac{\rho_{\mathrm{m}}}{2} h \mathcal{S}\langle V\rangle_{a}{ }^{2}+\frac{1}{2 \mu_{\mathrm{m}}}\left(\mathcal{B}\left\langle\Sigma_{1}\right\rangle_{a}{ }^{2}+\mathcal{C}\left\langle\Sigma_{2}\right\rangle_{a}{ }^{2}\right)\right] .
$$

We have used that $\mathcal{B}_{2}+\mathcal{C}_{1}=0$, and the proof is reported in Appendix A. Also, (44) has been obtained using integrations by parts, for instance of $\int \mathrm{d} X_{2} \partial_{X_{2}} V\left\langle\Sigma_{1}\right\rangle_{a}$; this makes additional terms at the upper and lower boundaries of $\mathcal{D}_{a}$ (along $X_{1}$ ) to appear and something should be said at these boundaries; this is disregarded in this paper. 
$\mathcal{E}_{a}$ is the energy associated to the equivalent interface; as previously said, $\mathcal{E}_{a}$ has to be positive in order to ensure that the homogenized problem is well suited for numerical purpose (the dependence of $\mathcal{E}_{a}$ on the thickness $a$ is in the parameters $(\mathcal{B}, \mathcal{C}, \mathcal{S})$ in $(44))$. In the following section, we inspect this condition with respect to the value of $a$.

\section{Optimal value of $a$ in the homogenized problem}

In this section, we inspect the influence of the thickness $a=O(e, h)$ in the homogenized problem. This is done first considering the equation of energy conservation, and it is shown that for $a \geq e$, the interface parameters $(\mathcal{S}, \mathcal{B}, \mathcal{C})$ entering in the definition of the energy supported by the interface are positive, thus ensuring $\mathcal{E}_{a} \geq 0$ in (44). Next, the error between the solution of the homogenized problem and the solution of the actual problem is considered; this is done in the particular scattering problem of a plane wave at oblique incidence on an array of rectangular inclusions, which leads to substantial simplifications. Notably, an easy procedure based on multimodal method can be used to calculate the effective parameters (see Appendix B).

3.1 Influence of $a$ on the energy $\mathcal{E}_{a}$ supported by the interface in the homogenized problem

From (44), the energy supported by the homogenized interface is guarantied to be positive if $(\mathcal{S}, \mathcal{B}, \mathcal{C})$ are positive. We shall see that this is guarantied if $a \geq e$.

\subsubsection{Minimum value of a ensuring $\mathcal{E}_{a} \geq 0$}

- An obvious parameter is $\mathcal{S}$ in 40 and it is easy to see that

$$
\mathcal{S} \geq 0, \quad \text { if } \quad \frac{a}{e} \geq \varphi\left(1-\frac{\rho_{\mathrm{i}}}{\rho_{\mathrm{m}}}\right) .
$$

Next, to find bounds for $\mathcal{B}$ and $\mathcal{C}$, we shall use variational formulations of (18), yielding to principles of energy minimization.

- The parameter $\mathcal{B}$ is defined in $\left(40, \mathcal{B}=\mathcal{B}_{0}+a / h\right.$, with $\mathcal{B}_{0}$ in $(19)$. We start with $0=\int_{\Omega_{\infty}} \mathrm{d} \mathbf{y} \operatorname{div}\left[\mu \boldsymbol{\nabla}\left(W^{(1)}+y_{1}\right)\right] \tilde{W}$, with $\tilde{W}$ and its derivatives being square integrable, continuous at the boundaries between the matrix and the inclusions, and periodic with respect to $y_{2}$; this defines the set of admissible (or trial) fields $\tilde{W}$. We get

$\int_{\Omega_{\infty}} \mathrm{d} \mathbf{y} \mu \nabla W^{(1)} \cdot \nabla \tilde{W}+\int_{\Omega_{\infty}} \mathrm{d} \mathbf{y} \mu \nabla \tilde{W} \cdot \mathbf{e}_{1}-\mu_{\mathrm{m}}\left[\tilde{W}\left(+\infty, y_{2}\right)-\tilde{W}\left(-\infty, y_{2}\right)\right]=0$ 
from which we define the energy

$\mathcal{E}(\tilde{W})=\frac{1}{2} \int_{\Omega_{\infty}} \mathrm{d} \mathbf{y} \mu|\nabla \tilde{W}|^{2}+\int_{\Omega_{\infty}} \mathrm{d} \mathbf{y} \mu \boldsymbol{\nabla} \tilde{W} \cdot \mathbf{e}_{1}-\mu_{\mathrm{m}}\left[\tilde{W}\left(+\infty, y_{2}\right)-\tilde{W}\left(-\infty, y_{2}\right)\right]$.

The field $W^{(1)}$ minimizes $\mathcal{E}$, whence $\mathcal{E}(\tilde{W}) \geq \mathcal{E}\left(W^{(1)}\right)$ for any admissible $\tilde{W}$. Now, we need to express $\mathcal{E}\left(W^{(1)}\right)$ as a function of $\mathcal{B}_{0}$. To do so, we use $\tilde{W}=$ $W^{(1)}$ in 46 , from which

$$
\int_{\Omega_{\infty}} \mathrm{d} \mathbf{y} \mu\left|\nabla W^{(1)}\right|^{2}+\int_{\Omega_{\infty}} \mathrm{d} \mathbf{y} \mu \nabla W^{(1)} \cdot \mathbf{e}_{1}-\mu_{\mathrm{m}} \mathcal{B}_{0}=0 .
$$

Starting from $0=\int_{\Omega} \mathrm{d} \mathbf{y} \operatorname{div}\left[\mu \boldsymbol{\nabla}\left(W^{(1)}+y_{1}\right)\right] y_{1}$, we get

$$
0=-\int_{\Omega} \mathrm{d} \mathbf{y} \boldsymbol{\nabla} W^{(1)} \cdot \mathbf{e}_{1}-\int_{\Omega} \mathrm{d} \mathbf{y} \mu+\int_{\partial \Omega} \mathrm{d} l \mu y_{1} \boldsymbol{\nabla}\left(W^{(1)}+y_{1}\right) \cdot \mathbf{n} .
$$

The two last integrals diverge, but it is easy to see that their sum is finite, with $\int_{\Omega} \mathrm{d} \mathbf{y} \quad \mu=2 \mu_{\mathrm{m}} y_{1}^{m}+e \varphi / h\left(\mu_{\mathrm{i}}-\mu_{\mathrm{m}}\right)$ and $\int_{\partial \Omega} \mathrm{d} l \mu y_{1} \boldsymbol{\nabla}\left(W^{(1)}+y_{1}\right) \cdot \mathbf{n}=2 y_{1}^{m}$. Thus, summing them and taking the limit $y_{1}^{m} \rightarrow+\infty$, we get

$$
\int_{\Omega_{\infty}} \mathrm{d} \mathbf{y} \mu \nabla W^{(1)} \cdot \mathbf{e}_{1}=\frac{e \varphi}{h}\left(\mu_{\mathrm{m}}-\mu_{\mathrm{i}}\right) .
$$

It is now sufficient to use (48)- 49 to get

$$
\begin{aligned}
\mathcal{E}\left(W^{(1)}\right) & =\frac{1}{2} \int_{\Omega_{\infty}} \mathrm{d} \mathbf{y} \mu\left|\nabla W^{(1)}\right|^{2}+\int_{\Omega_{\infty}} \mathrm{d} \mathbf{y} \mu \nabla W^{(1)} \cdot \mathbf{e}_{1}-\mu_{\mathrm{m}} \mathcal{B}_{0} \\
& =\frac{1}{2}\left[\frac{e \varphi}{h}\left(\mu_{\mathrm{m}}-\mu_{\mathrm{i}}\right)-\mu_{\mathrm{m}} \mathcal{B}_{0}\right] .
\end{aligned}
$$

and lower bounds of $\mathcal{B}_{0}$ are deduced

$$
\mathcal{B}_{0} \geq \frac{e \varphi}{h}\left(1-\frac{\mu_{\mathrm{i}}}{\mu_{\mathrm{m}}}\right)-\frac{2}{\mu_{\mathrm{m}}} \mathcal{E}(\tilde{W}),
$$

for any admissible field $\tilde{W}$ (and $\mathcal{E}(\tilde{W})$ defined in (47p). To get an explicit bound, we consider the admissible function $\tilde{W}\left(y_{1}\right)$ independent of $y_{2}$ and with $\tilde{W}\left(y_{1}<-e /(2 h)\right)=-b, \tilde{W}\left(-e /(2 h) \leq y_{1}<e /(2 h)\right)=2 b h y_{1} / e$ and $\tilde{W}\left(y_{1} \geq\right.$ $e /(2 h))=b$, and at least for the time being $b$ is a free parameter. From (47), we get

$$
\mathcal{E}(\tilde{W})=\alpha b^{2}+\beta b, \quad \text { with } \alpha=\frac{2 h}{e}\left[\varphi \mu_{\mathrm{i}}+(1-\varphi) \mu_{\mathrm{m}}\right], \quad \beta=2 \varphi\left(\mu_{\mathrm{i}}-\mu_{\mathrm{m}}\right) .
$$

With $\alpha>0$, the minimum of $\mathcal{E}(\tilde{W})$ is realized for $b=-\beta /(2 \alpha)$ yielding $\mathcal{E}(\tilde{W})=-\beta^{2} /(4 \alpha)$. The resulting lower bound for $\mathcal{B}_{0}$ reads

$$
\mathcal{B}_{0} \geq \frac{e \varphi}{h} \frac{1-\mu_{\mathrm{i}} / \mu_{\mathrm{m}}}{\varphi \mu_{\mathrm{i}} / \mu_{\mathrm{m}}+1-\varphi},
$$


and obviously at this stage, $\mathcal{B}_{0}$ can be negative. Now, the minimum value of $a$ which ensures that $\mathcal{B} \geq 0$ in 40 is given by

$$
\mathcal{B}=\frac{a}{h}+\mathcal{B}_{0} \geq 0, \quad \text { if } \quad \frac{a}{e} \geq \varphi \frac{\mu_{\mathrm{i}} / \mu_{\mathrm{m}}-1}{\varphi \mu_{\mathrm{i}} / \mu_{\mathrm{m}}+1-\varphi} \equiv F\left(\mu_{\mathrm{i}} / \mu_{\mathrm{m}}, \varphi\right) .
$$

- As for $\mathcal{B}$, we want to get a condition on $a$ in order to ensure $\mathcal{C} \geq 0$, with $\mathcal{C}$ defined in $(40)$ as a function of $\mathcal{C}_{0}$ (in $(33)$, with $(18)$ ). We shall proceed as previously, except that we shall consider here the dual variational problem. To do so, we define

$$
\boldsymbol{S}^{(2)} \equiv \mu \boldsymbol{\nabla}\left[W^{(2)}+y_{2}\right] .
$$

$\boldsymbol{S}^{(2)}$ is associated to the following variational problem. For any admissible field $\tilde{\boldsymbol{S}}$ being divergence free, with $\tilde{\boldsymbol{S}} . \mathbf{n}$ continuous at the boundaries between the matrix and the inclusions and $\tilde{\boldsymbol{S}} \rightarrow \mu_{\mathrm{m}} \mathbf{e}_{2}$ at $y_{1} \rightarrow \pm \infty$, we have

$$
\mathcal{E}^{*}(\tilde{\boldsymbol{S}}) \geq \mathcal{E}^{*}\left(\boldsymbol{S}^{(2)}\right), \quad \text { with } \mathcal{E}^{*}(\tilde{\boldsymbol{S}}) \equiv \frac{1}{2} \int_{\Omega_{\infty}} \mathrm{d} \mathbf{y} \mu\left[\frac{\tilde{\boldsymbol{S}}}{\mu}-\mathbf{e}_{2}\right]^{2}
$$

and it is easy to see that

$$
\mathcal{E}^{*}\left(\boldsymbol{S}^{(2)}\right)=-\frac{\mu_{\mathrm{m}}}{2} \mathcal{C}_{0}
$$

To get the above relation, we start with $0=\int_{\Omega_{\infty}} \mathrm{d} \mathbf{y} \operatorname{div}\left[\mu \boldsymbol{\nabla}\left(W^{(2)}+y_{2}\right)\right] W^{(2)}$, whence

$$
\begin{aligned}
0 & =\int_{\Omega_{\infty}} \mathrm{d} \mathbf{y} \mu\left|\nabla W^{(2)}\right|^{2}+\int_{\Omega_{\infty}} \mathrm{d} \mathbf{y} \mu \frac{\partial \tilde{W}}{\partial y_{2}} \\
& =\int_{\Omega_{\infty}} \mathrm{d} \mathbf{y} \mu\left[\frac{\boldsymbol{S}^{(2)}}{\mu}-\mathbf{e}_{2}\right]^{2}+\mu_{\mathrm{m}} \mathcal{C}_{0} .
\end{aligned}
$$

Now, a lower bound of $\mathcal{C}_{0}$ can be found, owing to

$$
\frac{\mu_{\mathrm{m}}}{2} \mathcal{C}_{0} \leq-\mathcal{E}^{*}(\tilde{\boldsymbol{S}})
$$

once an admissible field $\tilde{\boldsymbol{S}}$ has been chosen. We built such $\tilde{\boldsymbol{S}}$ being piecewise constant along $y_{1}$ and independent of $y_{2}$, with $\tilde{\boldsymbol{S}}\left(\left|y_{1}\right|<e /(2 h)\right)=c \mathbf{e}_{2}$ and $\tilde{\boldsymbol{S}}\left(\left|y_{1}\right| \geq e /(2 h)\right)=\mu_{\mathrm{m}} \mathbf{e}_{2}$, and the $c$-value minimizing $\mathcal{E}^{*}(\tilde{\boldsymbol{S}})$ is sought. We get

$$
\mathcal{E}^{*}(\tilde{\boldsymbol{S}})=\frac{e}{2 h}\left[\alpha c^{2}-2 c+\beta\right], \quad \text { with } \alpha=\frac{1-\varphi}{\mu_{\mathrm{m}}}+\frac{\varphi}{\mu_{\mathrm{i}}}, \beta=(1-\varphi) \mu_{\mathrm{m}}+\varphi \mu_{\mathrm{i}} .
$$

With $\alpha>0$, the minimum of $\mathcal{E}^{*}(\tilde{\boldsymbol{S}})$ is reached for $c=1 / \alpha$, and $\mathcal{E}^{*}(\tilde{\boldsymbol{S}})=$ $(\alpha \beta-1) / \alpha$. The corresponding lower bound is

$$
\mathcal{C}_{0} \geq-\frac{e}{h} \frac{\varphi(1-\varphi)\left(\mu_{\mathrm{i}} / \mu_{\mathrm{m}}-1\right)^{2}}{\varphi+(1-\varphi) \mu_{\mathrm{i}} / \mu_{\mathrm{m}}}
$$


and $\mathcal{C}_{0}$ is clearly not always positive. Now, from 40

$$
\mathcal{C}=\frac{a}{h}+\frac{e \varphi}{h}\left(\mu_{\mathrm{i}} / \mu_{\mathrm{m}}-1\right)+\mathcal{C}_{0}
$$

from which

$$
\mathcal{C} \geq 0 \quad \text { if } \quad \frac{a}{e} \geq-\varphi \frac{1-\mu_{\mathrm{i}} / \mu_{\mathrm{m}}}{\varphi+(1-\varphi) \mu_{\mathrm{i}} / \mu_{\mathrm{m}}}=-F\left(\mu_{\mathrm{i}} / \mu_{\mathrm{m}}, \varphi\right) .
$$

\subsubsection{Remarks on the bounds - The case $a=e$}

From what precedes, the interface energy (44) is positive if the thickness $a$ satisfies

$$
a \geq e \operatorname{Max}\left(\left|F\left(\mu_{\mathrm{i}} / \mu_{\mathrm{m}}, \varphi\right)\right|, \varphi\left(1-\rho_{\mathrm{i}} / \rho_{\mathrm{m}}\right)\right) .
$$

If we want this to be verified for any contrast $\mu_{\mathrm{i}} / \mu_{\mathrm{m}}$ and any $\varphi$, the condition becomes $a \geq e$ (because $F \leq 1$ reaches its maximum $F=1$ for $\varphi=1$ and $\mu_{\mathrm{i}} / \mu_{\mathrm{m}}=0$ ). In the case $a=e$, Eqs. (45), (50), (58) give

$$
\left\{\begin{array}{l}
\mathcal{S} \geq \frac{e}{h}\left(1-\varphi+\varphi \rho_{\mathrm{i}} / \rho_{\mathrm{m}}\right), \\
\mathcal{B} \geq \frac{e}{h} \frac{1}{\varphi \mu_{\mathrm{i}} / \mu_{\mathrm{m}}+1-\varphi}, \\
\mathcal{C} \geq \frac{e}{h} \frac{\mu_{\mathrm{i}} / \mu_{\mathrm{m}}}{\varphi+(1-\varphi) \mu_{\mathrm{i}} / \mu_{\mathrm{m}}},
\end{array}\right.
$$

which guaranty that $\mathcal{S}, \mathcal{B}$ and $\mathcal{C}$ are positive.

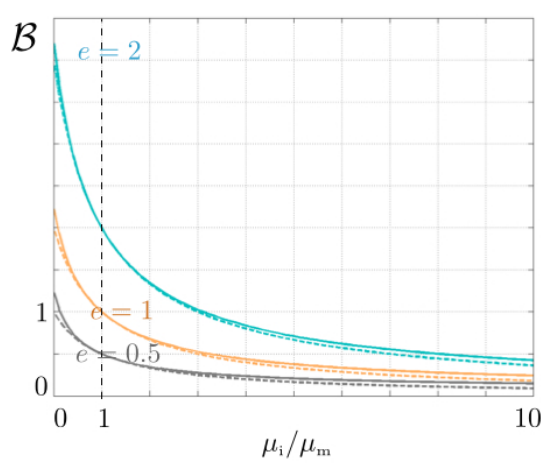

(a)

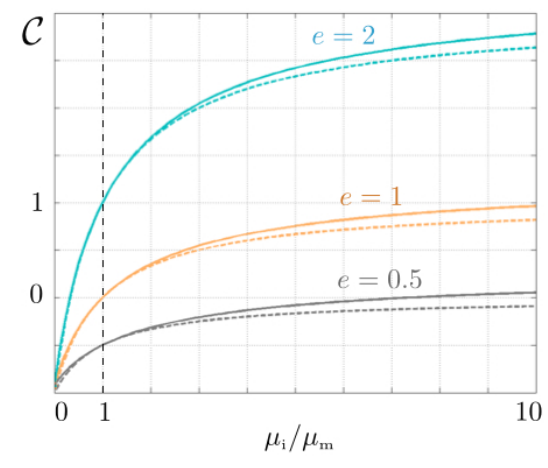

(b)

Figure 4 Interface parameters $\mathcal{B}$ and $\mathcal{C}$, Eqs. 40 , as a function of the contrast in the shear modulus $\mu_{\mathrm{i}} / \mu_{\mathrm{m}}$ for $e / h=0.5,1$ and 2 for $a=e$. Plain lines are the parameters calculated by solving $[18$, dotted lines are the bounds given by 60 .

For inclusions being rectangular voids or cracks, being the limiting case $\rho_{\mathrm{i}} / \rho_{\mathrm{m}}=\mu_{\mathrm{i}} / \mu_{\mathrm{m}}=0$, estimates of the parameters have been given in [1,2] (note 
that different notations are used in these references); with the notations used presently, they read

$$
\left\{\begin{array}{l}
\mathcal{S}_{N}=\frac{e}{h}(1-\varphi), \\
\mathcal{B}_{N} \simeq \frac{e}{h} \frac{1}{1-\varphi}+\frac{2}{\pi} \log \left(\sin \frac{\pi(1-\varphi)}{2}\right)^{-1} \\
\mathcal{C}_{N} \simeq \pi \frac{(1-\varphi)^{2}}{8}, \text { if } \frac{e}{h}<\frac{\pi(1-\varphi)}{8}, \quad \mathcal{C}_{N} \simeq 0, \text { otherwise }
\end{array}\right.
$$

and these values are in agreement with our bounds 60 for $\rho_{\mathrm{i}}=\mu_{\mathrm{i}}=0$; the value of $\mathcal{B}_{N}$ for $e=0$, which is always positive, has been obtained in [9] using technics of complex variables.

Finally, we report in Fig. 4 the variations of $\mathcal{B}$ and $\mathcal{C}$ as a function of the contrast $\mu_{\mathrm{i}} / \mu_{\mathrm{m}}$ (the parameters have been calculated using the scripts given in Appendix B for $\varphi=0.5)$. The bounds found in 60 are reported for comparison.

\subsection{Error of the model as a function of $a$}

In this section, we address the error of the homogenized solution when compared to the solution of the actual problem. This is done varying $a$, which affects the values of the effective parameters $(\mathcal{B}, \mathcal{C}, \mathcal{S})$ in the jump conditions (42), as it affects the size of the region $\mathcal{D}_{a}$ of the enlarged interface (Fig. 3). The error in the homogenized solution cannot be predicted within mathematical considerations and it has to be considered in a particular scattering problem; below, this is done in the case of a plane wave at oblique incidence on the array, which allows for an explicit homogenized solution. In the harmonic regime, the waves have a time dependence $e^{-\mathrm{i} \omega t}$ with frequency $\omega=2 \pi f$; in the following, we omit the time dependance with $V(\mathbf{X}, t)=V(\mathbf{X}) e^{-\mathrm{i} \omega t}$. We consider an incident wave, in $X_{1}<0$, written as

$$
V^{\mathrm{inc}}(\mathbf{X})=e^{i k\left(\cos \theta X_{1}+\sin \theta X_{2}\right)} \text {. }
$$

Regarding the material properties and inclusion shape, we shall consider rectangular fibers of steel in concrete, with $\rho_{\mathrm{i}}=7700 \mathrm{~kg} \cdot \mathrm{m}^{-3}, \mu_{\mathrm{i}}=78 \mathrm{GPa}$, $\rho_{\mathrm{m}}=2500 \mathrm{~kg} \cdot \mathrm{m}^{-3}$ and $\mu_{\mathrm{m}}=12 \mathrm{GPa}$. Note that for rectangular inclusions, being symmetric with respect to $y_{2}, W^{(1)}$ is symmetric and $W^{(2)}$ is antisymmetric with respect to $y_{2}$; it follows that $\mathcal{B}_{2}=\mathcal{C}_{1}=0$, in $(19)$ and $(33)$, and only $(\mathcal{S}, \mathcal{B}, \mathcal{C})$ are needed in the jump conditions.

Examples of the displacement fields in the harmonic regime are reported in Fig. 5 for $e / h=2, \varphi=0.5$, and frequency $f$ such that $k h=0.5$; typical configurations for such $k h$ value would correspond to centimetric sizes of the fibers in the kilohertz regime. In the real problem, the incident wave hitting the array generates an evanescent field. Although vanishing far from the fibers, the 
influence of this field cannot be neglected since it modifies the scattering properties of the propagating wave [2]. Thus a simple plane wave analysis would result in a high error in the real solution, and a full wave numerical method is required. We use a multimodal method (see e.g. [10]) and denote $V^{\text {num }}(\mathbf{X})$ the computed solution. It is worth noting that the direct numerics has been demanding, although the frequency is not high in the reported example (thus the difference between the wavelength and the fiber size is low); translating the number of modes considered in the numerics in terms of mesh size, the spatial step needed to get a converged solution was $\delta x / h=5 \cdot 10^{-3}$ and this is the price to pay to capture accurately the near field; a more detailed discussion on the requirement on the mesh size can be found in 2$]$ for calculations performed in the harmonic regime and in [1] for calculations performed in the time domain.

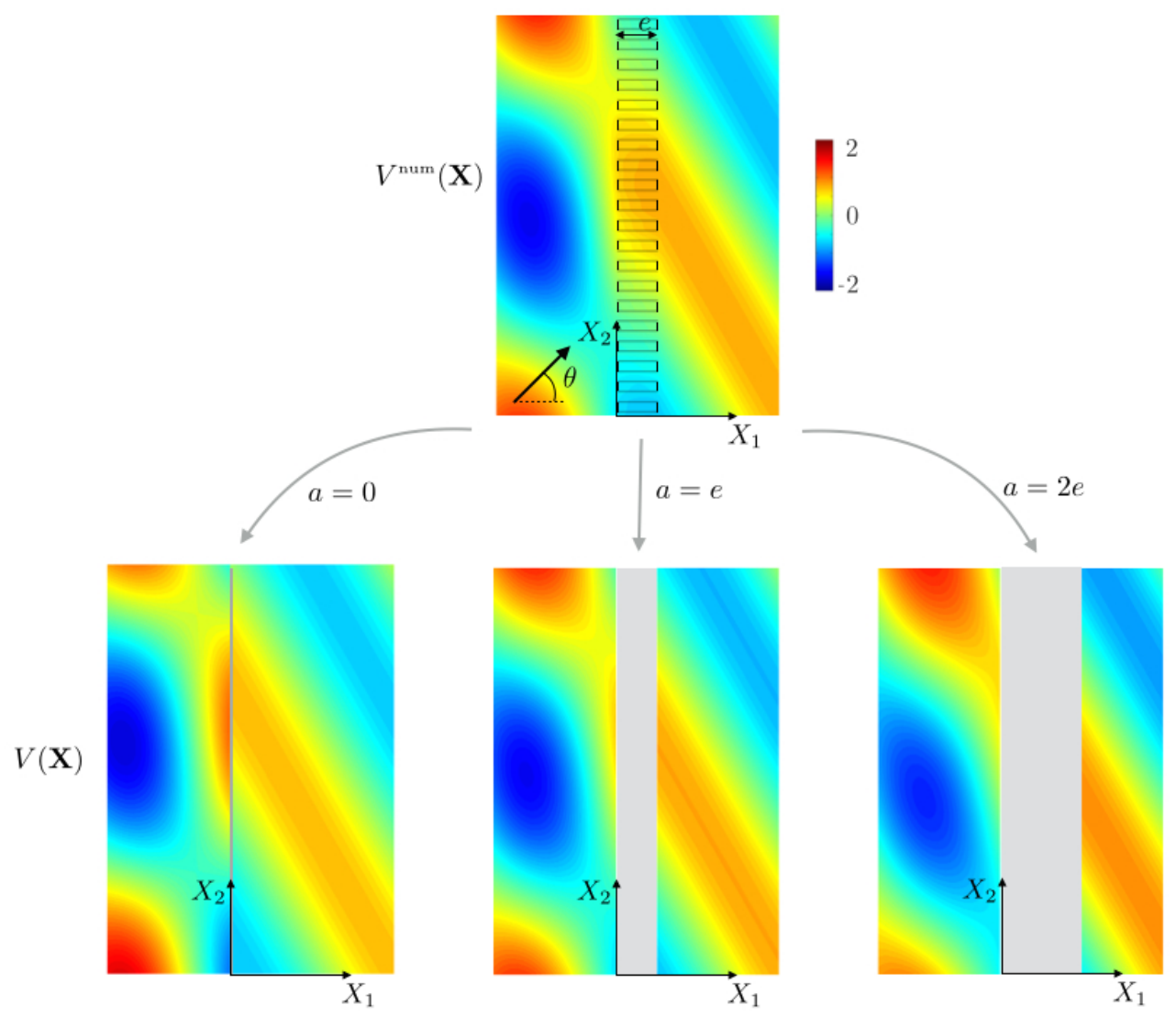

Figure 5 Fields $V^{\text {num }}(\mathbf{X})$ in the actual configuration of a matrix of concrete with fibers of steel. The dimensionless frequency is $k h=k e / 2=0.5$. The 3 fields $V(\mathbf{X})$ in the homogenized problem, Eqs. 62 - 63, for an equivalent interface of thickness $a=0, e$ and $2 e$. The discrepancy $E_{H^{1}}$, Eq. (64) for $X_{1}<0$, is $40 \%$ for $a=0,2 \%$ for $a=e$, and $30 \%$ for $a=2 e$. 
In the homogenized problem, the story is different; the boundaries of the equivalent interface are flat and the problem is one dimensional; thus, the solution is simply a superposition of plane waves, with

$$
V(\mathbf{x})= \begin{cases}e^{i k \sin \theta X_{2}}\left[e^{i k \cos \theta X_{1}}+R e^{-i k \cos \theta X_{1}}\right], & X_{1}<0, \\ e^{i k \sin \theta X_{2}} T e^{i k \cos \theta\left(X_{1}-a\right)}, & X_{1}>a,\end{cases}
$$

where the scattered waves $\left(V-V^{\text {inc }}\right)$ satisfy the radiation condition (outgoing waves are considered). Using 42 , the scattering properties $(R, T)$ are obtained explicitly, namely

$$
\left\{\begin{array}{l}
R=\frac{1}{2}\left[\frac{z_{2}^{*}}{z_{2}}-\frac{z_{1}^{*}}{z_{1}}\right], \quad T=\frac{1}{2}\left[\frac{z_{2}^{*}}{z_{2}}+\frac{z_{1}^{*}}{z_{1}}\right] \\
z_{1} \equiv 1-\frac{i k}{2} \mathcal{B} \cos \theta, \quad z_{2} \equiv \cos \theta-\frac{i k}{2}\left(\mathcal{S}-\mathcal{C} \sin ^{2} \theta\right)
\end{array}\right.
$$

where $z_{i}^{*}$ denotes the complex conjugate of $z_{i}$. The field $V$ is parametrized by $a$ since $(\mathcal{S}, \mathcal{B}, \mathcal{C})$ depend on $a$. Next, it differs from the solution that would be obtained using a plane wave analysis in the real problem. In fact, the influence of the evanescent field has been encapsulated in the interface parameters $(\mathcal{S}, \mathcal{B}, \mathcal{C})$; this is possible since for low frequency, the near field is essentially a static field (and loosely speaking, it is a combination of our elementary problems (18)).

In Fig. 5, we reported the solutions $V(\mathbf{X})$ of the homogenized problem, 62) with (63), for a zero thickness interface $a=0, e$ and $2 e$. The interface parameters 40 for $a=0$ are $: \mathcal{S}(a=0)=2.12, \mathcal{B}(a=0)=-1.39$ and $\mathcal{C}(a=0)=1.54$ (next, the values for $a=e$ are deduced by adding the factor $e / h=2$ to each parameter, and the values for $a=2 e$ are deduced by adding the factor $2 e / h=4$ to each parameter). Qualitatively, a very good agreement is observed for $a=e$, much better than for $a=0$ and $a=2 e$.

To be more quantitative, we define

$$
E_{H^{1}}=\frac{\left\|V-V^{\text {num }}\right\|_{H^{1}}}{\left\|V^{\text {num }}\right\|_{H^{1}}},
$$

the relative error in $H^{1}$ norm, with $\|V\|_{H^{1}} \equiv \sqrt{k^{2} V^{2}+(\nabla V)^{2}}$ and for simplicity, we consider the reflected field only (in $X_{1}<0$ ), for which no ambiguity on the computational domains occurs. Next, the error is calculated for $X_{1}<0$ which means that the evanescent near field is considered in addition to the propagating field or it is calculated for $X_{1}<-e$, thus interrogating the far field only. Results are reported in Fig. 6 for $a$ varying continuously in $[0,2 e]$ and for various $k h$ from $k h=10^{-3}$ to 1 . As expected, the error when including the near field is significantly higher than when considering only the far field. Indeed, the homogenized solution does not reproduce the evanescent field in the actual problem. This solution contains only a propagating field which depends of boundary layer problems encapsulated in the interface parameters. In the actual problem, the propagating field depends also on boundary layer 
effects encapsulated in the evanescent field (and the evanescent field occupies a region near the fibers).

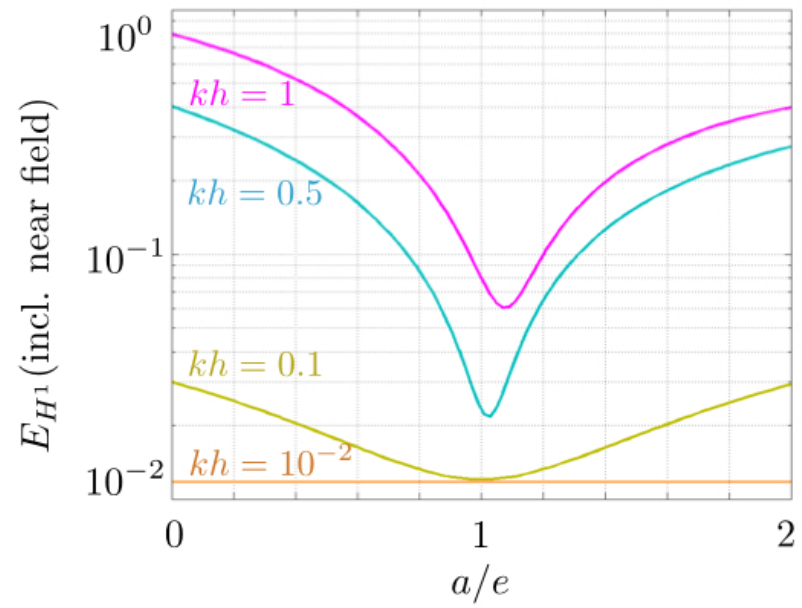

Figure 6 Discrepancy on the total field $E_{H^{1}}$, Eq. 64) calculated for $X_{1}<0$, as a function of a/e. The same configuration as in Fig. 5 has been considered, for various $k h$-values ( $k e=2 k h$ increases accordingly).

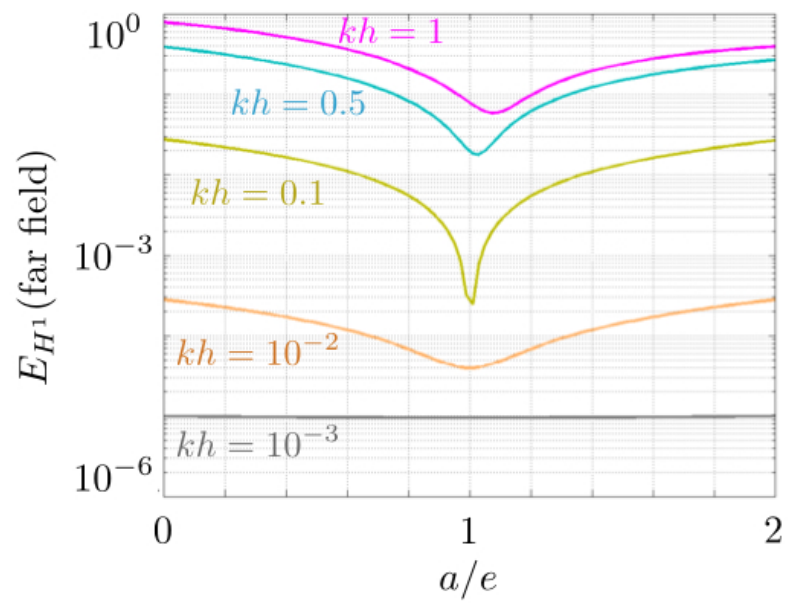

Figure 7 Discrepancy on the far field $E_{H^{1}}$ calculated for $X_{1}<-e$ (far field) as a function of $a / e$. The same configuration as in Fig. 5 has been considered, for various $k h$-values ( $k e=2 k h$ increases accordingly).

From Fig. 6, the $a$-value which produces the minimum of the error is at $a=e$; a small shift is observed for $k h$ close to unity which may happen for 
slightly smaller or larger value than $e$, with no systematic tendance (we observe here an increase in the optimum $a$-value while other contrast or size produce a decrease; in all cases, the shift appears for $k h$ close to 1$)$. The improvement in using $a=e$ is not incremental, with a decrease in the error on the scattering coefficients (Fig. 7) from typically 10 to 100 when compared to the case $a=0$ or $a=2 e$. Note that it is difficult to draw a tendency in the improvement as a function of $k h$ since for very small error, the error due to the numerical method may become dominant when compared to the error due to the model; besides, the norm $H_{1}$ requires a numerical derivation along $X_{1}$ in the direct numerics, which increases the numerical error (this is particularly true when the near field, with rapid variations, is considered).

\section{Concluding remarks}

We have presented an interface model to identify the effective behavior of an array of elastic inclusions embedded in an elastic matrix. Parameters characteristic of the interface enter in jump conditions for the displacement and the normal stress of the shear waves. These parameters are given by the resolution of elementary problems written in the static limit, and they are therefore wave independent by construction. We have inspected different forms of the jump conditions, being all equivalent up to the order of validity of the model and concluded that an equivalent interface being enlarged of the same thickness as the original array was a good choice to ensure that (i) the homogenized problem is well suited for numerical resolution (which means free of numerical instabilities in the time computation) and (ii) the error in the model is reduced when compared to the case of zero thickness interface (or other thickness of the homogenized interface). Obviously, the two questions are of different nature : if a positive interface energy is a prerequisite for any consistent computational method in the time domain (and as so, it is not optional), a low error in the model is only a wishful thinking.

\section{Références}

1. Marigo, J.-J. , Maurel, A. : Homogenization models for thin rigid structured surfaces and films. J. Acoust. Soc. Am. 140(1), 260-273 (2016)

2. Marigo, J.-J., Maurel, A. : An interface model for homogenization of acoustic metafilms, unpublished (2016).

3. Maurel, A., Marigo, J.-J., Ourir, A. : Homogenization of ultrathin metallo-dielectric structures leading to transmission conditions at an equivalent interface. J. Opt. Soc. Am. 33(5) 947-956 (2016).

4. Marigo, J.-J., Maurel, A. : Two scale homogenization to determine effective parameters of thin metallic structured films. Proc. R. Soc. A. 472(2192) 20160068 (2016).

5. Delourme, B., Haddar, H. , Joly, P. : Approximate models for wave propagation across thin periodic interfaces. J. Math. Pures Appl. 98, 28-71 (2012)

6. Delourme, B. : High-order asymptotics for the electromagnetic scattering by thin periodic layers. Math. Meth. Appl. Sciences, 38(5) 811-833 (2015) 
7. Marigo, J.-J., Pideri, C. : The effective behavior of elastic bodies containing microcracks or microholes localized on a surface. Int. J. Damage Mech. 1056789511406914 (2011)

8. David, M., Marigo, J. J., Pideri, C. : Homogenized interface model describing inhomogeneities located on a surface. Journal of Elasticity. 109(2), 153-187 (2012).

9. Morse, P. M., Ingard, K. U. : Theoretical acoustics, Princeton university press, (1968)

10. Maurel, A., Mercier, J.-F., Félix, S. : Wave propagation through penetrable scatterers in a waveguide and through a penetrable grating. J. Acoust. Soc. Am. 135(1), 165-174 (2014)

11. Lombard, B., Maurel, A., Marigo, J.-J. : Numerical modeling of the acoustic wave propagation across an homogenized rigid microstructure in the time domain, unpublished (2016)

\section{A Proof of the relation $\mathcal{B}_{2}+\mathcal{C}_{1}=0$ used in 44}

We recall the definitions of $\mathcal{B}_{2}$ and $\mathcal{C}_{1}$ :

$$
\mathcal{B}_{2}=W^{(2)}\left(+\infty, y_{2}\right)-W^{(2)}\left(-\infty, y_{2}\right), \quad \mathcal{C}_{1}=\int_{\Omega_{\infty}} \mathrm{d} \mathbf{y} \frac{\mu(\mathbf{y})}{\mu_{\mathrm{m}}} \frac{\partial W^{(1)}}{\partial y_{2}}
$$

with $W^{(\mathrm{i})}$ satisfying $[18$, and we will show that

$$
\mathcal{B}_{2}=-\mathcal{C}_{1}=\int_{\Omega_{\infty}} \mathrm{d} \mathbf{y} \frac{\mu(\mathbf{y})}{\mu_{\mathrm{m}}} \nabla W^{(1)} \nabla W^{(2)} .
$$

We start with

$$
\begin{aligned}
& 0=\int_{\Omega} \mathrm{d} \mathbf{y} \operatorname{div}\left[\mu \boldsymbol{\nabla}\left(W^{(1)}+y_{1}\right)\right] W^{(2)} \\
& =-\int_{\Omega} \mathrm{d} \mathbf{y} \mu \boldsymbol{\nabla}\left(W^{(1)}+y_{1}\right) \nabla W^{(2)}+\int_{\partial \Omega} \mathrm{d} l \mu \partial_{n}\left(W^{(1)}+y_{1}\right) W^{(2)}
\end{aligned}
$$

The second integral in $(70)$ simplifies because of (i) the continuity of $\mu \partial_{n}\left(W^{(1)}+y_{1}\right)$ and of $W^{(2)}$ on $\partial \Omega_{\mathrm{i}}$ and (ii) the periodicity of $W^{(\mathrm{i})}$ with respect to $y_{2}$. Only the integrals on the boundaries at $y_{1}= \pm y_{1}^{m}$ give a contribution, whence

$$
\int_{\partial \Omega} \mathrm{d} l \mu \partial_{n}\left(W^{(1)}+y_{1}\right) W^{(2)}=\mu_{\mathrm{m}}\left[W^{(2)}\left(y_{1}^{m}\right)-W^{(2)}\left(-y_{1}^{m}\right)\right] \rightarrow \mu_{\mathrm{m}} \mathcal{B}_{2}
$$

where the arrow indicates the limit for $y_{1}^{m} \rightarrow+\infty$ (we also used that $\partial_{y_{1}} W^{(1)}$ vanishes at $\left.y_{1} \rightarrow \pm \infty\right)$. The first integral in 70 reads $\int_{\Omega} \mathrm{d} \mathbf{y} \mu \boldsymbol{\nabla}\left(W^{(1)}+y_{1}\right) \nabla W^{(2)}=\int_{\Omega} \mathrm{d} \mathbf{y} \mu \boldsymbol{\nabla} W^{(1)} \nabla W^{(2)}+$ $\int_{\Omega} \mathrm{d} \mathbf{y} \mu \nabla W^{(2)} . \mathbf{e}_{1}$ and we shall show that $\int_{\Omega_{\infty}} \mathrm{d} \mathbf{y} \mu \nabla W^{(2)} \cdot \mathbf{e}_{1}=0$. To do so, we use that

$0=\int_{\Omega_{\infty}} \mathrm{d} \mathbf{y} \operatorname{div}\left[\mu \boldsymbol{\nabla}\left(W^{(2)}+y_{2}\right)\right] y_{1}=-\int_{\Omega_{\infty}} \mathrm{d} \mathbf{y} \mu \boldsymbol{\nabla} W^{(2)} \cdot \mathbf{e}_{1}+\int_{\partial \Omega_{\infty}} \mathrm{d} l \mu \partial_{n}\left(W^{(2)}+y_{2}\right) y_{1}$.

Again, the integral over $\partial \Omega_{\infty}$ simplifies, but now it simply vanishes. Coming back to 70 , we get $\mathcal{B}_{2}=\lim _{y_{1}^{m} \rightarrow+\infty} \int_{\Omega} \mathrm{d} \mathbf{y} \mu \nabla W^{(1)} \nabla W^{(2)}$.

We now inspect $\mathcal{C}_{1}$; we use

$$
\begin{aligned}
& 0=\int_{\Omega} \mathrm{d} \mathbf{y} \operatorname{div}\left[\mu \boldsymbol{\nabla}\left(W^{(2)}+y_{2}\right)\right] W^{(1)} \\
& =-\int_{\Omega} \mathrm{d} \mathbf{y} \mu \boldsymbol{\nabla}\left(W^{(2)}+y_{2}\right) \nabla W^{(1)}+\int_{\partial \Omega} \mathrm{d} l \mu \partial_{n}\left(W^{(2)}+y_{2}\right) W^{(1)} .
\end{aligned}
$$


It is easy to see that the second integral vanishes when $y_{1}^{m} \rightarrow+\infty$, and we simply get

$$
0=-\int_{\Omega_{\infty}} \mathrm{d} \mathbf{y} \mu \nabla W^{(1)} \nabla W^{(2)}-\int_{\Omega_{\infty}} \mathrm{d} \mathbf{y} \mu \frac{\partial W^{(1)}}{\partial y_{2}},
$$

which allows us to conclude.

\section{B Solutions of the elementary problems}

Mode matching is a simple way to solve the elementary problem for rectangular inclusions. The basic idea is to expand the solution onto an basis being adapted in each part of the unit cell. The solutions are periodic with respect to $y_{2}$, and for $\left|y_{1}\right|>e / 2$, the adapted transverse functions are simply

$$
f_{n}^{+}\left(y_{2}\right)=e^{2 \mathrm{i} n \pi y_{2}}
$$

which are orthogonal for the usual scalar product

$$
(f, g)_{1} \equiv \int \mathrm{d} y f(y) g^{*}(y)
$$

with $g^{*}$ the complex conjugate of $g$. For $\left|y_{1}\right| \leq e / 2$, an adapted basis $f_{n}^{0}$ has to be found, and adapted means here that the $f_{n}^{0}$ have to be periodic and have to satisfy the boundary condition at $y_{2}= \pm \varphi / 2,\left(f_{n}^{0}\right)^{\prime}\left(\varphi / 2^{-}\right)=a_{0}\left(f_{n}^{0}\right)^{\prime}\left(\varphi / 2^{+}\right)$(the same at $\left.y_{2}=-\varphi / 2\right)$. It is easy to see that the $f_{n}^{-}$are orthogonal for the scalar product

$$
(f, g)_{2}=\int_{|y| \leq \varphi / 2} \mathrm{~d} y f(y) g(y)+\xi \int_{|y|>\varphi / 2} \mathrm{~d} y f(y) g(y) .
$$

Next, the elementary solution will be expressed as a function of a field $V(\mathbf{y})$ written in a generic form

$$
V(\mathbf{y})= \begin{cases}V^{-}(\mathbf{y})=L(\mathbf{y})+\sum_{n=-N^{-}}^{N^{-}} q_{n}^{-} e^{b_{n}\left(y_{1}+e / 2\right)} f_{n}^{+}\left(y_{2}\right), & y_{1} \leq-e / 2, \\ W(\mathbf{y})=L(\mathbf{y})+\sum_{n=1}^{N}\left[W_{n}^{-} e^{b_{n}\left(y_{1}-e / 2\right)}+W_{n}^{+} e^{-b_{n}\left(y_{1}+e / 2\right),}\right] f_{n}^{-}\left(y_{2}\right), & \left|y_{1}\right| \leq e / 2 \\ V^{+}(\mathbf{y})=L(\mathbf{y})+\sum_{n=-N^{+}}^{N^{+}} q_{n}^{+} e^{-b_{n}\left(y_{1}-e / 2\right)} f_{n}^{+}\left(y_{2}\right), & y_{1} \geq e / 2,\end{cases}
$$

with $L(\mathbf{y})$ the loading being defined piecewise.

Now, we will ask to $V^{-}$and $W$ to match (on average) their values and their first derivatives at $y_{1}=-e / 2$ (same for $V^{+}$and $W$ at $y_{1}=e / 2$ ). To that aim, we use the following continuity relations at $\left|y_{1}\right|=e / 2$

at $y_{1}=-e / 2\left\{\begin{array}{l}\left(V^{-}, f_{m}^{+}\right)_{1}=\left(W, f_{m}^{+}\right)_{1}, \\ \left(\frac{\partial V^{-}}{\partial y_{1}}, f_{m}^{-}\right)_{1}=\left(\frac{\partial W}{\partial y_{1}}, f_{m}^{-}\right)_{2},\end{array}\right.$ at $y_{1}=e / 2\left\{\begin{array}{l}\left(W, f_{m}^{+}\right)_{1}=\left(V^{+}, f_{m}^{+}\right)_{1}, \\ \left(\frac{\partial W}{\partial y_{1}}, f_{m}^{-}\right)_{2}=\left(\frac{\partial V^{+}}{\partial y_{1}}, f_{m}^{-}\right)_{1} .\end{array}\right.$.

The matching conditions on the derivatives of $\left(V^{ \pm}, W\right)$ w.r.t. $y_{1}$ naturally account (on average) the exact conditions

$$
\left\{\begin{array}{l}
\frac{\partial W}{\partial y_{1}}\left(e / 2, y_{2}\right)=\frac{\partial V^{+}}{\partial y_{1}}\left(e / 2, y_{2}\right), \quad\left|y_{2}\right| \leq \varphi / 2 \\
\frac{\partial W}{\partial y_{1}}\left(e / 2, y_{2}\right)=\frac{1}{\xi} \frac{\partial V^{+}}{\partial y_{1}}\left(e / 2, y_{2}\right),\left|y_{2}\right|>\varphi / 2
\end{array}\right.
$$


(3) provide a matrix system for the vectors $\mathbf{W}^{ \pm}=\left(W_{n}^{ \pm}\right)_{n=1, \ldots, N}$ and $\mathbf{V}^{ \pm}=\left(V_{n}^{ \pm}\right)_{n=0, \ldots, N^{ \pm}}$ of the form

$$
\left(\begin{array}{cccc}
\mathrm{l} & 0 & -\mathrm{FE} & -\mathrm{F} \\
-{ }^{t} \mathrm{~F}^{*} \mathrm{~B} & 0 & \mathrm{BE} & -\mathrm{B} \\
0 & -1 & \mathrm{~F} & \mathrm{FE} \\
0 & { }^{t} \mathrm{~F}^{*} \mathrm{~B} & \mathrm{~B} & -\mathrm{BE}
\end{array}\right)\left(\begin{array}{l}
\mathbf{V}^{-} \\
\mathbf{V}^{+} \\
\mathbf{W}^{+} \\
\mathbf{W}^{-}
\end{array}\right)=\mathbf{S}
$$

with I the $N^{-} \times N^{-}$identity matrix, B $\equiv \operatorname{diag}(|2 n \pi|), \mathrm{E} \equiv \operatorname{diag}\left(e^{-|2 n \pi| e}\right)$. The matrix $F_{m n} \equiv\left(f_{n}^{0}, f_{m}\right)_{1}$ depend on the basis $f_{n}^{0}$ and the source term $\mathbf{S}$ depends on the loading $L(\mathbf{y})$; it is worth nothing that the above system has to be written accounting for the symmetry of the problem, which is accounted for in the choice of the $\left(f_{n}, f_{n}^{0}\right)$. The system is of the form $\mathbf{M q}=\mathbf{s}$ with the matrix $\mathrm{M}$ being square (this is not always the case in systems written using mode matching). Next, $\mathrm{M}$ is invertible if one has taken care to consider the correct symmetry. Thus, the system can be solved to find $\mathbf{q}$ by inverting $M$ or it can be solved in the least squares sense (as done by the operation $\mathrm{M} \backslash \mathbf{s}$ in Matlab).

In general, the $f_{n}^{0}$ have to be determined numerically. An exception for $\varphi=1 / 2$ is given below.

B.1 $\mathcal{B}_{0}$ in the elementary $W^{(1)}$ problem

For $\varphi=1 / 2$, a simple adapted basis $f_{n}^{-}$for symmetric solutions is

$$
f_{n}^{-}\left(y_{2}\right)= \begin{cases}A \cos \left(2 n \pi y_{2}\right), & n \text { even } \\ B \cos \left(2 n \pi y_{2}\right) \times \begin{cases}1, & \left|y_{2}\right|>1 / 4, \\ \xi, & \left|y_{2}\right| \leq 1 / 4,\end{cases} & n \text { odd }\end{cases}
$$

with $A \equiv 2 / \sqrt{1+\xi}, B=2 / \sqrt{\xi+\xi^{2}}$. We get the matrix $\mathrm{F}$

$$
F_{m n}=\left\{\begin{array}{lll}
A\left[g_{m n}+G_{m n}\right], & n \text { even, } \\
B\left[\xi g_{m n}+G_{m n}\right], & n \text { odd }
\end{array}\right.
$$

with

$$
\left\{\begin{array}{l}
g_{m n}=\frac{1}{4}\left[\operatorname{sinc} \frac{(n-m) \pi}{2}+\operatorname{sinc} \frac{(n+m) \pi}{2}\right], \\
G_{m n}=\frac{1}{2}[\operatorname{sinc}((n-m) \pi)+\operatorname{sinc}((n+m) \pi)]-2 g_{m n} .
\end{array}\right.
$$

The basis $\left(f_{n}, f_{n}^{0}\right)$ are adapted for $V=W^{(1)}+y_{1}$ satisfying $\Delta V=0$ in each medium, with $V$ with $a \boldsymbol{\nabla} V . \mathbf{n}$ being continuous ; next, $V \rightarrow \mathbf{e}_{1}$ for $y_{1} \rightarrow \pm \infty$, which gives the loading

$$
L\left(\left|y_{1}\right|>e / 2, y_{2}\right)=y_{1}, \quad L\left(\left|y_{1}\right| \leq e / 2\right)=\frac{2 y_{1}}{a_{0}+1} .
$$

The form of $L$ for $\left.\left|y_{1}\right| \leq e / 2\right)$ is chosen to ensure that the integral form of the equation $\operatorname{div}\left(a \boldsymbol{\nabla} W^{(1)}\right)=0$, namely $\int d y_{2} a(\mathbf{y}) \partial W^{(1)} / \partial y_{1}=1$, is satisfied. The resulting source term $\mathbf{S}$ reads ${ }^{t} \mathbf{S}={ }^{t}\left(\mathbf{S}_{\mathbf{1}}, \mathbf{S}_{\mathbf{2}}, \mathbf{S}_{\mathbf{1}}, \mathbf{S}_{\mathbf{2}}\right)$ with $\mathbf{S}_{\mathbf{1}}$ a $2 N^{ \pm}+1$ term vector and $\mathbf{S}_{\mathbf{2}}$ a $N$ term vector

$$
\left(S_{1}\right)_{n}=\frac{e}{2} \frac{1-\xi}{1+\xi} \delta_{n 0}, \quad\left(S_{2}\right)_{n}=B \frac{\xi-1}{2} \operatorname{sinc}\left(\frac{n \pi}{2}\right),
$$

with $\operatorname{sinc} x \equiv \sin x / x($ and $\operatorname{sinc} 0=0)$. The system 78 can be solved and $\mathcal{B}$ is given by

$$
\mathcal{B}_{0}=V_{0}^{+}-V_{0}^{-} \text {. }
$$


B.2 The elementary $W^{(2)}$ problem

Here, we consider $V=W^{(2)}+y_{2}$ which satisfies $\Delta V=0$ in each medium, with $V$ and $a \boldsymbol{\nabla} V \mathbf{n}$ being continuous at each interface and $V \rightarrow y_{2}$ for $y_{1} \rightarrow \pm \infty$ ( $V-y_{2}$ is periodic). The solution being now antisymmetric w.r.t. $y_{2}$, a simple adapted basis is

$$
f_{n}^{-}\left(y_{2}\right)= \begin{cases}A \sin \left(2 n \pi y_{2}\right), & n \text { odd } \\
B \sin \left(2 n \pi y_{2}\right) \times\left\{\begin{array}{ll}
1, & \left|y_{2}\right|>1 / 4, \\
\xi, & \left|y_{2}\right| \leq 1 / 4,
\end{array} \quad n \neq 0\right. \text { even }\end{cases}
$$

with the same normalizations $(A, B)$. It follows that $F_{m n}=\left(f_{n}^{0}, f_{m}\right)_{1}$ is defined as in 80 , but now

$$
\left\{\begin{array}{l}
g_{m n}=\frac{1}{4 \mathrm{i}}\left[\operatorname{sinc} \frac{(n-m) \pi}{2}-\operatorname{sinc} \frac{(n+m) \pi}{2}\right], \\
G_{m n}=\frac{1}{2 \mathrm{i}}[\operatorname{sinc}((n-m) \pi)-\operatorname{sinc}((n+m) \pi)]-g_{m n},
\end{array}\right.
$$

For $n=0, f_{0}^{0}\left(y_{2}\right)$ is a linear (periodic) function of $y_{2}$

$$
f_{0}^{0}\left(y_{2}\right)=\frac{1-\xi}{1+\xi} \begin{cases}y_{2}-1 / 2, & y_{2}>1 / 4 \\ -y_{2}, & \left|y_{2}\right| \leq 1 / 4 \\ y_{2}+1 / 2, & y_{2}<-1 / 4\end{cases}
$$

In this case, the loading contains not only the term $y_{2}$ but also $f_{0}^{0}\left(y_{2}\right)$ and this is to ensure that the term in $V$ linear in $y_{2}$ satisfies the right boundary condition at $\left|y_{2}\right|=1 / 4$ for $e \rightarrow 0\left(V_{n}^{ \pm}\right.$and $W_{n}^{ \pm}$vanish $)$; it is worth noting that setting $W_{0}=1$ is possible since $V$ is defined up to a constant. Thus

$$
L\left(\left|y_{1}\right|>e / 2, y_{2}\right)=y_{2}, \quad L\left(\left|y_{1}\right| \leq e / 2, y_{2}\right)= \begin{cases}y_{2}, & \left|y_{2}\right|>1 / 4 \\ y_{2}+f_{0}^{0}\left(y_{2}\right) & \left|y_{2}\right| \leq 1 / 4\end{cases}
$$

resulting in a source term ${ }^{t} \mathbf{S}={ }^{t}\left(\mathbf{S}_{\mathbf{1}}, \mathbf{0}, \mathbf{S}_{\mathbf{1}}, \mathbf{0}\right)$ with $\mathbf{S}_{\mathbf{1}}$ a $2 N^{ \pm}+1$ term vector

$$
\left(S_{1}\right)_{m}=\left(f_{0}^{0}, f_{m}\right)_{1}=\frac{\xi-1}{\xi+1} \frac{\operatorname{sinc}(m \pi / 2)}{2 \mathrm{i} m \pi} .
$$

Once the problem is solved, we want to determine

$$
\mathcal{C}_{0}=\int \mathrm{d} \mathbf{y} a(\mathbf{y}) \frac{\partial W^{(2)}}{\partial y_{2}}=\int_{-e / 2}^{e / 2} \mathrm{~d} y_{1}\left(1, \frac{\partial W}{\partial y_{2}}-1\right)_{2}
$$

where we have used that $a W^{(2)}\left(\left|y_{1}\right| \geq e / 2, y_{2}\right)=V^{ \pm}(\mathbf{y})-y_{2}$ is periodic, thus of vanishing contribution. It results

$$
\mathcal{C}_{0}=\frac{1-\xi}{2}\left[-\frac{1-\xi}{1+\xi} e+A \sum_{n=1}^{N}\left(W_{n}^{-}+W_{n}^{+}\right) \operatorname{sinc} \frac{n \pi}{2}\left(1-e^{-2 n \pi e}\right)\right]
$$

\section{B.3 Matlab scripts}

Below, we give the scripts to calculate $\mathcal{B}_{0}$ and $\mathcal{C}_{0}$ in Matlab. 


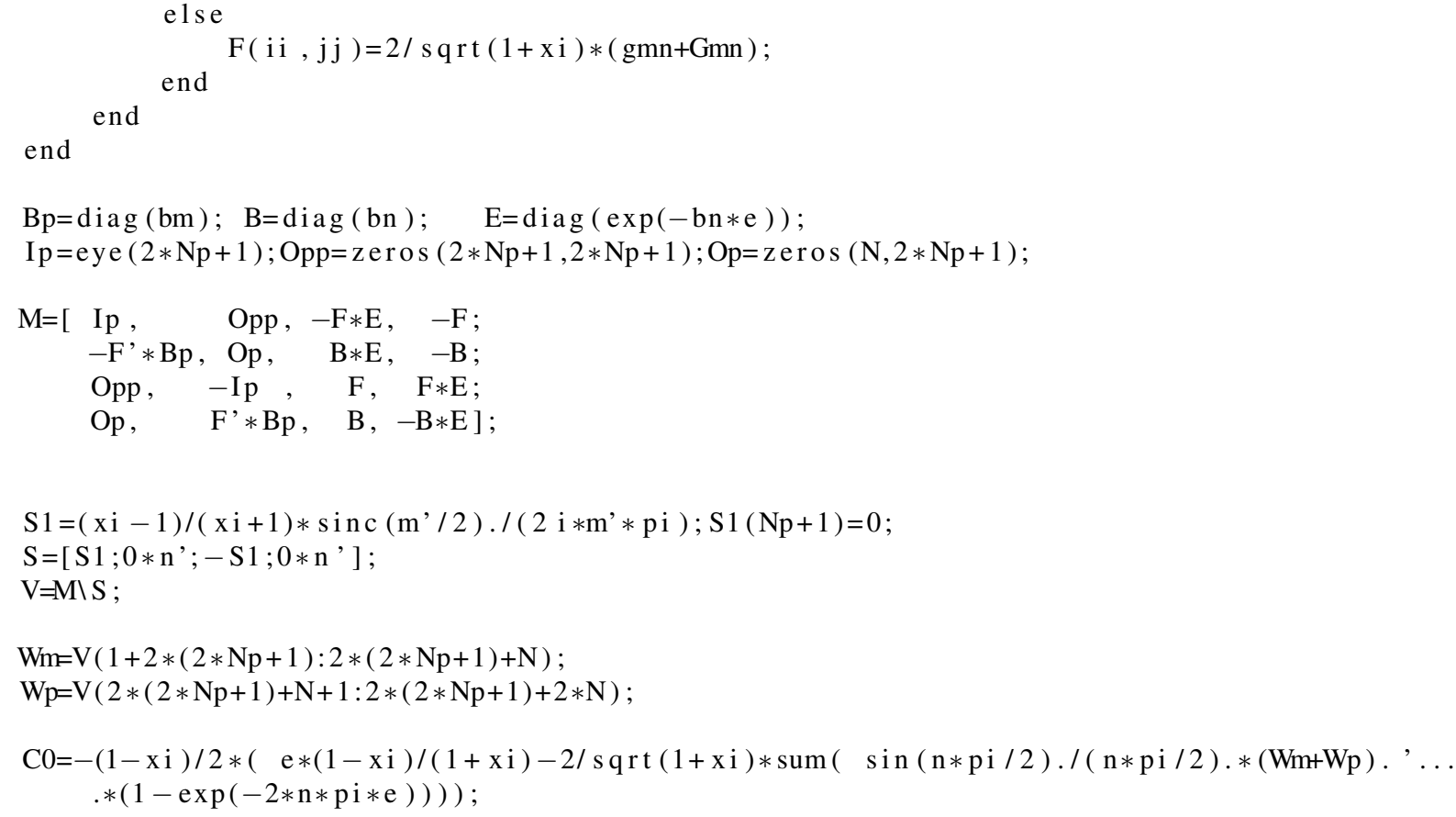

